\title{
IDENTIFYING RELATIONSHIP BETWEEN PATIENT ACUITY INDICATORS AND NURSE WORKLOAD
}

\author{
A Thesis presented to \\ the Faculty of the Graduate School \\ at the University of Missouri \\ In Partial Fulfillment \\ of the Requirements for the Degree \\ Master of Science, Industrial Engineering
}

by

BAYRAM DUNDAR

Dr. Mustafa Sir, Thesis Supervisor

JULY 2013 
The undersigned, appointed by the Dean of the Graduate School, have examined the thesis entitled:

\title{
IDENTIFYING RELATIONSHIP BETWEEN PATIENT ACUITY INDICATORS AND NURSE WORKLOAD
}

\author{
Presented by Bayram Dundar, \\ A candidate for the degree of Master of Science, \\ And hereby certify that, in their opinion, it is worthy of acceptance.
}

Dr. Mustafa Sir

Dr. Kalyan Pasupathy

Dr. Esra Sisikoglu 


\section{ACKNOWLEDGMENTS}

I would like to appreciate all those who provided me the possibility to complete this thesis. First, I would like to acknowledge my deepest gratitude to Dr. Mustafa Sir, who was not only an advisor, but also a friend. Dr. Sir, thank you for your unique insight and support that has been an outstanding source of my knowledge and strength.

I extend my thanks to the members of my thesis committee: Dr. Esra Sisikoglu, Dr. Kalyan Pasupathy. I would like to thank you, Dr. Sisikoglu, for your valuable suggestions and providing encouragement and guidance. Thank you Dr. Kalyan for your unique contribution to my thesis. I want to extend my gratitude to Dr. Linsey Barker Steege whose guidance and support was precious.

I would like to thank my wife, Ruveyda, who has supported me throughout entire process, both by giving me motivation and helping me persist. I will be grateful forever for your love.

I want to extend my gratitude to my colleague, Esma, for her Python programming assistance. I also would like to give a special thank to Dr. Kemal Izci, for his help as reviewer of my thesis.

My thanks and appreciations also go to my family and my wife's family who have emotionally supported me and have always given me motivation and strength.

I would like to extend my gratitude to Turkish Ministry of National Education (MEB) for providing a long term financial support during my master degree in the United States. Thank you for supporting Turkish students abroad to make their dreams come true. 


\section{TABLE OF CONTENTS}

ACKNOWLEDGMENTS ................ ii

LIST OF FIGURES $\ldots \ldots \ldots \ldots \ldots \ldots \ldots$ v

LIST OF TABLES $\ldots \ldots \ldots \ldots \ldots \ldots$ vii

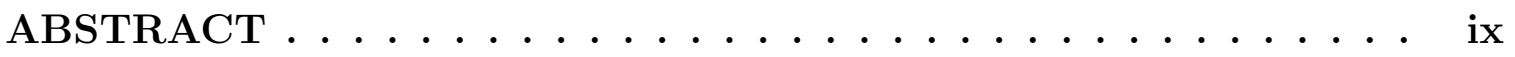

\section{CHAPTER}

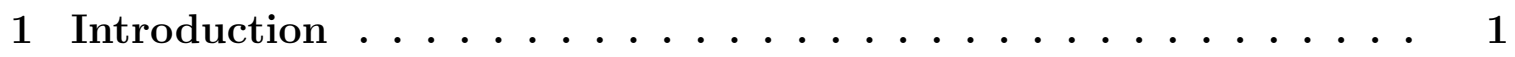

2 Literature Review . . . . . . . . . . . . . . 4

2.1 Work Analysis Methodologies . . . . . . . . . . . . . . 4

2.2 Traditional Optimization Models in Nurse Planning _ . . . . . . 10

2.3 Patient Classification Systems . . . . . . . . . . . . . . . 12

3 Data Collection and Analysis . . . . . . . . . . . 17

3.1 Analysis of Patient Acuity Indicator Data $\ldots \ldots \ldots$

3.2 Nurse Workload Survey . . . . . . . . . . . . . . . . . . . . . . . . . 21

4 Nurse-Patient Assignment Problem . . . . . . . . . . . . 28

4.1 Nurse-Patient Assignment Model with an Objective of Balancing Patient Acuity Scores from AcuityPlus PCS . . . . . . . . . . . . 29

4.2 Nurse-Patient Assignment Model Based on Balancing Survey-Based Workload

4.3 Nurse-Patient Assignment Model Based on Minimizing Total SurveyBased Workload . . . . . . . . . . . . . . . . . . . 
4.4 Nurse-Patient Assignment Model Based on Minimizing Maximum Survey-Based Workload . . . . . . . . . . . . . . . . 34

5 Numerical Results . . . . . . . . . . . . . . 36

5.1 Discussion . . . . . . . . . . . . . . . . . . 42

6 Conclusion and Future Works . . . . . . . . . . . . 44 APPENDIX

A Health Sciences Institutional Review Board Approval Letter . . 46

B Survey............................ 49

C Python Code ....................... 53

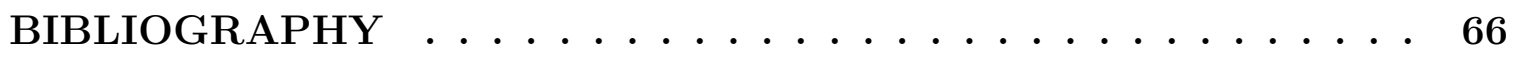




\section{LIST OF FIGURES}

Figure

2.1 Link analysis for a registered nurse during a shift. Frequency of lines between two locations show how many times the nurse moves between them $($ Potter et al. 2004) $\ldots \ldots \ldots \ldots \ldots$. . . . . . . . 7

2.2 Cognitive Pathway for a registered nurse (Potter et al. 2004) . . . . 8

3.1 Trend of average patient acuity score based on the thirteen week period for both oncology and surgery nursing units $\ldots \ldots \ldots \ldots$

3.2 Percentage of patients who has a specific patient acuity indicator . . 19

3.3 The average scores of patient acuity indicators based on the survey. The number on the $\mathrm{x}$-axis are the indices of the patient acuity indicators, the corresponding names of which are given in Table $2.3 \ldots$.

3.4 Survey-based average perceived workload of each patient acuity indicators for oncology and surgery nursing units. . . . . . . . . . . 24

3.5 The standard deviation of survey-based average perceived workload of each patient acuity indicators for the oncology and surgery nursing

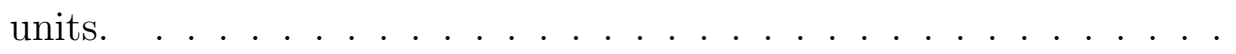


3.6 The relationship between the survey-based perceived workload and the patient type from AcuityPlus data. . . . . . . . . . . 26

3.7 The relationship between the survey-based perceived workload and the complexity type of patient from the AcuityPlus data. . . . . . . 26

5.1 Boxplot comparing the mean perceived workload for different models 39

5.2 Distribution of the mean perceived workload for different models . . 40

5.3 Boxplot comparing the standard deviation perceived workload for different models . . . . . . . . . . . . . . . . . . . . 41

5.4 Distribution of the standard deviation perceived workload for different

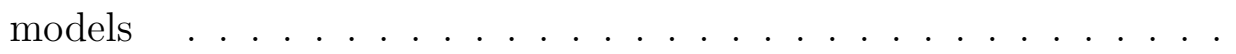




\section{LIST OF TABLES}

Table $\quad$ Page

2.1 AcuityPlus methodology patient types (QuadraMed 2008) . . . . . . 14

2.2 AcuityPlus inpatient complexity of care (QuadraMed 2008) . . . . . . 15

2.3 AcuityPlus inpatient classification indicators with their recorded number in the AcuityPlus systems (QuadraMed 2008) . . . . . . . . . 16

3.1 The example of patients that have the same acuity score but very different acuity indicator distributions. . . . . . . . . . . . . . . 20

3.2 Patient information recorded in the AcuityPlus database obtained from the University of Missouri Hospital. . . . . . . . . . . . . . . 21

3.3 Likert scale used in nurse workload survey . . . . . . . . . . . . 22

3.4 The experience distribution of nurses . . . . . . . . . . . . . 23

3.5 Statistical analysis of patient acuity indicators based on nurses' survey result. . . . . . . . . . . . . . . . . . . . . . 27

5.1 Notations used in the remaining chapters for models described in Chap-

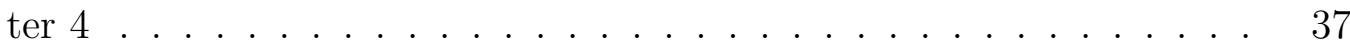

5.2 ANOVA results comparing the mean perceived workload of the four nurse-patient assignment model described in Chapter 4 . . . . . . . 
5.3 Result of the LSD method comparing the mean perceived workload for the four assignment models. The difference in the mean perceived workload for the models in the same group is not statistically significant. 38

5.4 ANOVA results comparing the standard deviation perceived workload of the four nurse-patient assignment model described in Chapter 4. . 40

5.5 Result of the LSD method comparing the standard deviation perceived workload for the four assignment models. The difference in the standard deviation perceived workload for the models in the same group is not statistically significant. . . . . . . . . . . . . . . . 


\title{
IDENTIFYING RELATIONSHIP BETWEEN \\ PATIENT ACUITY INDICATORS \\ AND NURSE WORKLOAD \\ Bayram Dundar \\ Dr. Mustafa Sir, Thesis Supervisor
}

\begin{abstract}
Patient classification systems are commonly used in nursing units to assess how many nursing care hours are needed to care for patients. In current practice, these systems utilize several patient acuity indicators to produce a single number for given patient, which is called the acuity score. These systems then provide staffing recommendations for a given patient census based on these acuity scores. Our new approach offers a mathematical model which uses patient acuity indicators data from a commercial patient classification system called AcuityPlus and a nurse workload survey from two nursing units at the University of Missouri Hospital. The model distributes workload calculated as a function of patient acuity indicators and nurses' perceived workload among nurses in a balanced way. More specifically, we consider nurses' preferences by conducting a survey in order to determine how much the workload of a nurse increases for each indicator. The purpose of this study is to conduct a comprehensive analysis of patient acuity indicators as a mean of balancing workload of nurses, and suggest nurse staffing and assignment models to aid decision-making. Our numerical results suggest that the proposed nurse-patient assignment models achieve a more balanced assignment of workload among nurses compared to assignment based on acuity scores from the commercial patient classification system.
\end{abstract}




\section{Chapter 1}

\section{Introduction}

Human factors engineering methods have been widely used in various industries such as aviation, manufacturing, and healthcare (Wolf et al. 2006). Due to the increased costs and difficult work conditions, researchers have been paying more attention to healthcare related research to make the healthcare environment more efficient. The significant part of health care personnel cost belongs to nursing expenditure, which is about $40 \%$ of general personnel cost (Mullinax et al. 2002). Therefore, it is important to focus on work environment of nurses to increase their satisfaction and decrease healthcare cost by increasing efficiency. According to a survey conducted by the American Nurses Association (2011), 74 percent of nurses highlighted stress and overwork as a main concern while 62 percent emphasized musculoskeletal injuries. According to Bureau of Health professions, the shortage of full-time equivalent registered nurses is expected to be 808,406 nurses by the year of 2020 (Biviano et al. 2005). Therefore, it is critical to increase satisfaction of nurses to retain them in nursing care.

As reported by Battisto et al. (2009), the reasons why nurses leave their position or 
job include personnel and safety concerns, performing complex job responsibilities containing medication administration, navigating documentation systems, working in an inefficient environment, and musculoskeletal injuries.

These surveys or previous researches conclude that if the problems associated to nurse work environment are not addressed by managers, the shortage of nurses will become a major problem in healthcare very soon. (Ebright et al. 2003) claimed that eighty three percent of nurses agree that improving nurses' environment prevents nurse shortage. Therefore, it is critical for human factors engineers, health care professionals, and industrial engineers to collaborate in order to develop innovative ways to improve nursing environment to prevent this catastrophic situation from occurring.

Nursing work analysis methods highlighted in the human factor engineering literature such as task analysis, link analysis, cognitive pathway, and stacking can help human factors engineers to understand the requirements of nursing job, what nurses are doing, how often they do an activity or a task, the workload of nurses, the complications caused by interventions, cognitive shifts, and etc. In addition, using various optimization techniques, industrial engineers can develop models to develop better work schedules and improve working conditions of nurses by balancing workload among nurses. Furthermore, there are only a few previous studies that incorporate human factors into optimization model for improving nurses work conditions. For example, Lin et al. (2013) incorporated quantified models of fatigue into a nurse scheduling model considering nurse shift preference and nurse fatigue. In this thesis, we developed a nurse-patient assignment model with objective of balancing workload among nurses in order to improve their work conditions. The workload used in our 
assignment model is based on a survey conducted in two nursing units at the University of Missouri hospital. The survey is conducted with an aim to determine how much each patient acuity indicator increases the workload of a nurse. The survey allows us incorporating nurses' perceived workload into the assignment model, which results a better workload distribution among nurses. We compare the performance of our proposed assignment model with an assignment model based on acuity scores from the commercial patient classification system.

The remainder of the thesis is outlined as follows. In Chapter 2, we review work analysis methodolgies, tradional optimization models, and patient classification system. In Chapter 3, we present analysis of patient acuity indicator data and nurse workload survey. In Chapter 4, we develop three new integer programming models for the nurse-patient assignment problem. We solve this model for two nursing units in an academic hospital and analyze four nurse-patient assignment model in Section 5. Finally, in Section 6, we offer conclusions and directions for future works. 


\section{Chapter 2}

\section{Literature Review}

\subsection{Work Analysis Methodologies}

In this section, some of the relevant human factor methods including task analysis, link analysis, cognitive pathway, stacking and interruption used in our study are reviewed to provide background for this research.

Task Analysis: In nursing, it is important to know detailed steps, or activities so as to analyze work environment. Task analysis is one of the nursing analysis methods that allows experts to investigate elaborated individual steps or activities and classify sub-tasks in a nursing environment (Battisto et al. 2009). Battisto et al. (2009) used task analysis in order to understand and improve nurse' work conditions. Using task analysis, nurses activities can be followed regularly so as to decide how to help them ameliorating their work conditions. For nurses, knowing what they will do, which sequence they are going to perform, or how much time they should spend on a 
certain task can make their job easier. As was mentioned earlier, nurses quit their job because of complex job responsibilities. Therefore, classification of certain activities or steps into groups or sub-tasks can aid nurses to easily comprehend complex tasks and thus encourage them to retain their jobs. In addition, this classification can also prevent medical error in terms of patients, and to ameliorate nurses work condition.

Battisto et al. (2009) used three main research methods in order to observe and classify nurses activities and priorities. These methods include 1) examination of nursing education, 2)observational research, and 3) structured interviews. The approach used in their study asks some crucial questions to describe the task analysis. These questions are fundamental for conducting a task analysis for any kind of job. The exemplary questions to describe the task analysis in their research include;

1. What are the activities that nurses typically carry out in an acute care patient room?

2. What are the frequencies of these activities?

3. Where do these activities typically occur?

4. What environmental problems are encountered while performing these activities?

After observing nurses and interviewing them, they developed a taxonomy of nursing activities table. In addition, they analyzed several metrics such as the percentage of occurrences of each task, percentage of visits to nursing task locations, and usage frequency of equipment item used to provide a comprehensive picture for nurses work environment. With the ample knowledge of detailed steps and sub-steps of nurse tasks, the nurse work environment can be improved. Task analysis can be effectively 
used to determine the workload of a nurse for a given assignment. These workload models can be easily incorporated into the nurse-patient assignment models described in Chapter 4.

Link analysis: Link analysis is another nurses' work analysis method to investigate motion of nurses between certain points where nurses stop by during their shift (Potter et al. 2004). This analysis method provides researchers the frequency of the motions between each location during patient caring process, which can then be used to eliminate the unnecessary movements that increase nurses' fatigue and impact patient satisfaction. Wolf et al. (2006) provided a link analysis example, which is depicted in Figure 2.1. The figure summarizes a registered nurse's movement during a shift. Their example contains eleven patient rooms, two pantries, two nurses station, medication room, and lounge where nurse visited during working shift. Dark lines in Figure 2.1 show how many times the nurse visited these locations. For instance, there are two lines between the patient $1 \mathrm{~b}$ and $1 \mathrm{a}$, which means the nurse traveled between the patient $1 \mathrm{~b}$ and 1a twice. The nurse-patient assignment methods developed in Chapter 4 do not explicitly incorporate nurse movements into the objective function and constraints. Link analysis can be used either directly incorporating nurse movement as an additional consideration into the assignment model or indirectly as a post-analysis to improve assignments.

Cognitive Pathway analysis: Cognitive pathway method defined by Wolf et al. (2006) employs both qualitative and quantitative methods to analyze nurses' work conditions The cognitive pathway method shows cognitive shifts of a nurse during an observed time. For example, Figure 2.2 shows the cognitive pathway of a nurse who is taking care of 6 patients. Each entry on the vertical axis represents a patient. The 


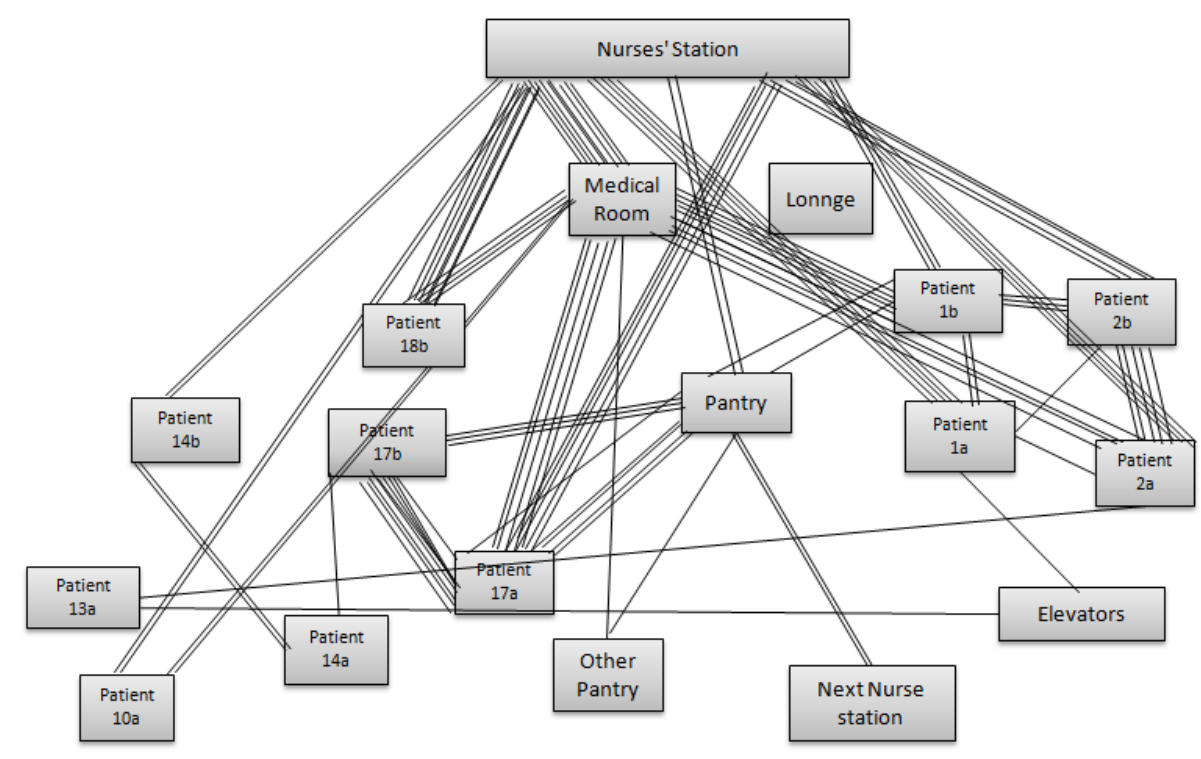

Figure 2.1: Link analysis for a registered nurse during a shift. Frequency of lines between two locations show how many times the nurse moves between them (Potter et al. 2004)

horizontal axis at the top of the figure is the time and the one on the bottom shows the interruptions.

The interruptions are identified using Nurse Caring Process (NCP). NCP is a standardized approach used by a nurse during the patient caring process to determine, elicit, and evaluate patient's response to cure (Potter et al. 2004). NCP includes five main steps:

1. Assessment: inquire the initial and ongoing condition of inpatients.

2. Diagnosis: define patients' condition and priorities.

3. Planning: includes plans related to discharge and consultation with patients and other staff members contributing to caring process. 


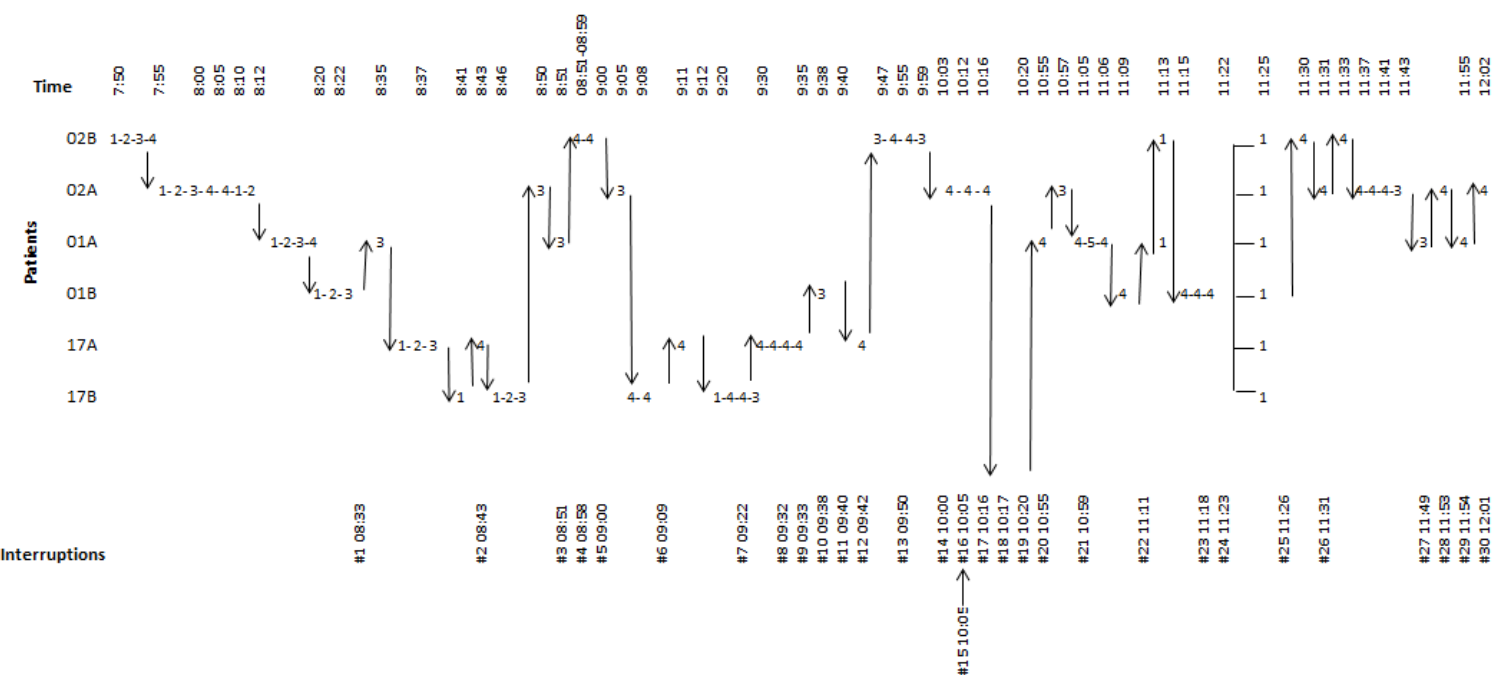

Figure 2.2: Cognitive Pathway for a registered nurse (Potter et al. 2004)

4. Intervention: this step covers direct and indirect care measures, such as documentation and medication preparation.

5. Evaluation: analyzing the response of patient to treatment.

By following these steps, an observer records related data to form the cognitive pathway. As seen in Figure 2.2, registered nurse follows these steps in sequence. For example, for patient 02B, the observed nurse follows steps 1-2-3-4 during caring process. Then, both a cognitive and physical shift happens in order to care for the next patient 02A. The interruptions are defined to be the shifts seen on these cognitive pathways and they can be caused by either physical movement or cognitive shift or both during the caring process. For instance, an interruption is recorded due to a cognitive shift when the nurse is interrupted by a staff in order to assess another patient's condition at time 08:43. The reason why cognitive pathway is important for researchers is because of the map created after observed shift provides important information of how many times nurses change their attention from one 
process to another and the frequency of interruptions during a shift. In fact, this kind of information can be used to improve nurses' work conditions and eliminate negative patient outcomes. Detailed analysis of several cognitive pathways can also help researchers to determine more useful objective functions and constraints for nurse-patient assignment models.

Stackings and Interruptions: The number of stackings and interruptions are other crucial parameters in order to analyze nurses' work conditions. Wolf et al. (2006) define the stacking as a group of activities or priorities that nurses are expected to perform for assigned patients.Patterson et al. (2011) based on interviewed registered nurses to inquire how they prioritize their activities. In their research, they found that stacking is a critical performance criterion as it shows the workload of nurses. Therefore, the workload of nurses should be also considered before a shift while a head nurse or managers try to assign certain number of nurses to inpatients in order to improve the work conditions. Furthermore, when the number of accumulated activities or priorities in the stacking increase, it impacts the nurse performance in a negative way which increases the job complexity. As mentioned earlier, complex job responsibilities negatively impact the job satisfaction of nurses, which in turn makes them quit their jobs (Kalisch \& Aebersold 2010). That is, developing assignment models based on stacking that measures workload ca be effective in enhancing nurses work conditions.

In this chapter, we reviewed several human factors methods, which give us a detailed map of the nursing process. Each of these methods separately might provide different information about workload of nurses. For instance, using link analysis, we can calculate average motion of nurses during a shift and then convert it a corre- 
sponding patient nursing time. In the remainder, we only consider patient acuity indicators to calculate nurses workload. However, it must be noted that the number of interruptions occurred during a shift and the motions of nurses between patients or other places such as nurses' station, pantry, and medical room must also be considered ta be able to accrately estimate nurse workload. That is why, besides patient acuity indicators, analytic results the aforementioned human factors methods will be considered in future work.

\subsection{Traditional Optimization Models in Nurse Plan- ning}

While assignment is among the classical optimization, the nurse-patient assignment problems hos not been extensively studied in the operation research literature. On the other hand, nurses scheduling or rostering problems (Aickelin \& Li 2007, Maenhout \& Vanhoucke 2010, 2013, Bilgin et al. 2012) and nurse budgeting have been widely studied to improve working condition and therefore satisfaction of nurses (Sundaramoorthi et al. 2009). The ultimate aim of nurse scheduling problems is to assign nurses to certain shifts to decrease health cost, negative patient outcomes, and improve nurse satisfaction (Rais \& Viana 2011).

In this thesis, we develop nurse-patient assignment models to balance nurse workload. One of the major contributions of our study is to incorporate patient acuity indicators and nurses' perceived workload while assigning nurses to patients. It is important to note that while patient indicators are very useful in classifying patients and their needs for care, they fail to capture how each nurse perceives their workload 
increases for a given patient with various indicators. For example, a nurse may be more experience with surgery patients and be more comfortable handling indicators, which typically exist in surgery patients, whereas another nurse might prefer caring for patients with different indicators. Therefore, the perceived workload increased in for a given indicators would be different for each nurse. To analyze this, we conducted a survey among nurses from oncology and surgery nursing units at the University of Missouri hospital and asked how nurses perceive the increase in their workload for various indicators.

Sundaramoorthi et al. (2009) used a simulation approach to evaluate the nursepatient assignments using a real data set. In this study, tree-based models and kernel density estimation(KDE)methods were utilized as data mining tools to extract information from data to develop a simulation model for nurse activities. The result is a decision support system that helps head nurses to make a better decision for promoting patient care, balancing workload of nurses, reducing hospitals cost, and retaining nurses. In a follow-up study, Sundaramoorthi et al. (2010) developed a data-integrated simulation based optimization method for assigning nurses to patients prior to a shift. In addition to prior to shift assignments, they also consider assignment of patient admitted during a shift based on two heuristic-based policies with a goal of minimizing the differences in workload among nurses.

Recently, Punnakitikashem et al. (2013) focused on the nurse staffing and assignment problem with an objective of balancing workload. They developed a stochastic integer programming model to minimize excess workload by considering a hard budget constraint. In addition, They used several variants of the method as the solution approach. In another study Hertz \& Lahrichi (2008) developed a mixed integer pro- 
gramming model to assign patients to nurses for home care services. They used a Tabu Search heuristic to solve the problem which includes non-linear constraints and objectives. In a particularly relevant study to this thesis, Mullinax et al. (2002) developed a detailed patient acuity system to quantify the nurse workload in a neonatal intensive care unit. The authors incorporated this patient acuity system into an integer program that assigns each nurse to a group of patients to balance the workload.

Identifying appropriate nurse-patients ratios is another approach to ameliorate nurses' work condition and improving quality of patient safety and care. In the literature, there have been several studies to determining appropriate nurse-patient ratios (Kortbeek et al. 2012, Wright et al. 2006, de Véricourt \& Jennings 2011, Yankovic \& Green 2011, 2008). For instance, de Véricourt \& Jennings (2011) developed a closed queuing model to determine efficient nurse staffing policies. They consider a medical unit, which contains of $s$ homogeneous nurse and $n$ homogeneous patients, as a closed $M / M / s / / n$ queuing system. In their analysis, they identified effective staffing policies, which differ from the classic patient-nurse ratios used in practice. In another study Yankovic \& Green (2011) show that inflexible nurse-patient ratios cause either understaffing or overstaffing. They also claiming that unit size, nursing intensity, and unit length of stay impact nurses' work performance.

\subsection{Patient Classification Systems}

Patient classification systems (PCS) have been widely used to determine how many nursing hours a patient needs in order to being cared. According to Brennan et al. (2012), patient acuity tools have been utilized for quantifying nursing care needs for 
patients since the early 1960. Harper \& McCully (2007) also note that patient classification systems help managers to estimate required number of nursing staff and to accurately determine nurse-patient ratio. In addition, they indicate that patient classification systems are beneficial for improving patient outcomes, controlling health care systems' budgets, and nurse retention. Since patients have different acuity levels, they are classified in a variety of types. On the other hand, California Bill AB 394, which fixes patient-nurse assignment ratio as mentioned by (de Véricourt \& Jennings 2011), is criticized by many researchers due to its weaknesses in fairly considering acuity level of patients and distributing workload of nurse. Since patient classification systems provide detailed knowledge about patients through various patient activity indicators, they cab be effectively used while assigning nurses to patients in an equitable manner.

In this study, we used a rich data set from a commercial patient classification system called AcityPlus developed by the QuadraMed company. QuadraMed is AcuityPlus provides many functionalities to aid decision-making related and manage nursing resources efficiently (Graf et al. 2003). In AcuityPlus, patients are categorized into one of six types based on the patient indicators as seen in Table 2.1. Based on our discussion with a head nurse at university of Missouri hospital, we learned that this classification system has been used at the university hospital for about twenty years for managing nursing resources.

AcuityPlus utilizes two approaches namely task oriented and process oriented in order to construct patient classifications to determine required staffing. The workload of nurses in the task oriented approach is defined by multiplying task frequency with 


\begin{tabular}{|c|c|c|c|c|c|c|}
\hline Type & 1 & 2 & 3 & 4 & 5 & 6 \\
\hline Acuity & 0.7 & 1.0 & 1.5 & 2.3 & 3.1 & 4.6 \\
\hline $\mathrm{NCH} / 24$ & $0-4$ & $4-7$ & $7-10$ & $10-14$ & $14-20$ & 20 \\
\hline Points & $0-17$ & $18-36$ & $37-56$ & $57-79$ & $80-104$ & 105 \\
\hline
\end{tabular}

Table 2.1: AcuityPlus methodology patient types (QuadraMed 2008)

the standard time of tasks. After determining the workload, the system suggests the required number of nurses. In the process-oriented approach, workload index takes patient needs for care into account to suggest staffing requirements. Nurse-patient assignments are based on the process-oriented approach consisting of two translation steps. In the first step, workload index or census and acuity from patient classification outputs are identified, and then in the second step by using the census and acuity outputs, suggestions are provided for assignments (QuadraMed 2008). In addition, complexity type of patient is considered as another criteria while applying nurse-patient assignment process. Complexity types are determined by considering inpatient complexity score and their points to make decision to categorize patients within one of the five complexity types for equitably distributing nurses' workload as seen in Table 2.2. In the next section, we explain how this complexity type scores do not convey important knowledge about a patient while assigning nurses to patients.

When using AcuityPlus, nurses base their classification on their observation, assessment, cueing, and intervation. Observation is a recording of an event or situation by some mechanical devices or anyone. For instance, a telemetry monitor records pa- 


\begin{tabular}{|l|c|c|c|c|c|}
\hline Type & 1 & 2 & 3 & 4 & 5 \\
\hline $\begin{array}{l}\text { Complexity } \\
\text { Score }\end{array}$ & 1.0 & 2.0 & 3.0 & 4.0 & 5.0 \\
\hline Points & $0-26$ & $27-31$ & $32-40$ & $41-49$ & $50+$ \\
\hline
\end{tabular}

Table 2.2: AcuityPlus inpatient complexity of care (QuadraMed 2008)

tients' cardiac rhythm. Assessment is another classification process that is required professional judgment. In this process, necessary information is obtained by a professional care provider to evaluate patient's physiological and psychological situation. Cueing is completing an activity by considering verbal and visual guidance. The last classification process is intervention, which is done based on information from the assessment process. After one or some of above mentioned processes are completed, a patient can be categorized using the AcuityPlus inpatient classification indicators listed in Table 2.3. In the next section, we develop a workload model based on these indicators and survey results.

Detailed information about patient classification indicators given in the table 2.3 can be found in the user manual of the AcuityPlus system (QuadraMed 2008). The manual provides the definition of each indicator and examples of appropriate and inappropriate applications. 


\begin{tabular}{|ll|}
\hline 1-ADL - Self/Minimal Care & 14-Physiological Assessment - q 4 Hours \\
2-ADL - Partial Care & 15-Physiological Assessment - q 2 Hours \\
3-ADL - Complete Care & 16-Physiological Assessment - q 1 Hour \\
4-ADL - Rehabilitative & 17-Physiological Assessment - 30 minutes \\
5-ADL Assistance - 2-3 Caregivers & 18-Medication Preparation $\geq 20$ Minutes \\
6-ADL-Assistance-4 or More Caregivers & 19-Wound/Injury Management \\
7-Communication Support & 20-Wound/Injury Mgmt $\geq 30$ Minutes \\
8-Cognitive Support & 21-Healthcare Mgmt Education $\geq 1$ Hour \\
9-Behavior/Emotional Management & 21-Healthcare Mgmt Education $\geq 1$ Hour \\
10-Behavior/Emotional Mgmt - q 1 Hour & 46-PtOb/confusion \\
11-Safety Management - q 2 Hours & 48-PtOb/mental illness \\
12-Safety Management - q 30 Minutes & 50-PtOb/suicide precautions \\
13-Isolation Precautions (Transmission-Based) & 70-PtOb/fall risk \\
\hline
\end{tabular}

Table 2.3: AcuityPlus inpatient classification indicators with their recorded number in the AcuityPlus systems (QuadraMed 2008) 


\section{Chapter 3}

\section{Data Collection and Analysis}

\subsection{Analysis of Patient Acuity Indicator Data}

This section represents a comprehensive analysis of patient acuity data recorded in the AcuityPlus PCS obtained from oncology and surgery units of the University of Missouri Hospital in order to support the rationale of our research. This is a retrospective study using patient acuity indicators and recommended actual staff data to decide how to optimally assign patients to nurses. The data was collected from January 1, 2013 until April 9, 2013 from the University of Missouri Health System to determine a new nurse-patient assignment policy incorporating the impact of patient classification indicators on workload assessed by nurses. This study includes

all patients in the oncology and surgery units and survey results from the actual staff caring for these patients. To prevent creating any bias in the collected data, we did not exclude any patients or nurses from the survey. The response rate for the survey is 
approximately 80\%. The existing data in this study includes 2865 and 3241 patients for the oncology and surgery nursing units, respectively.

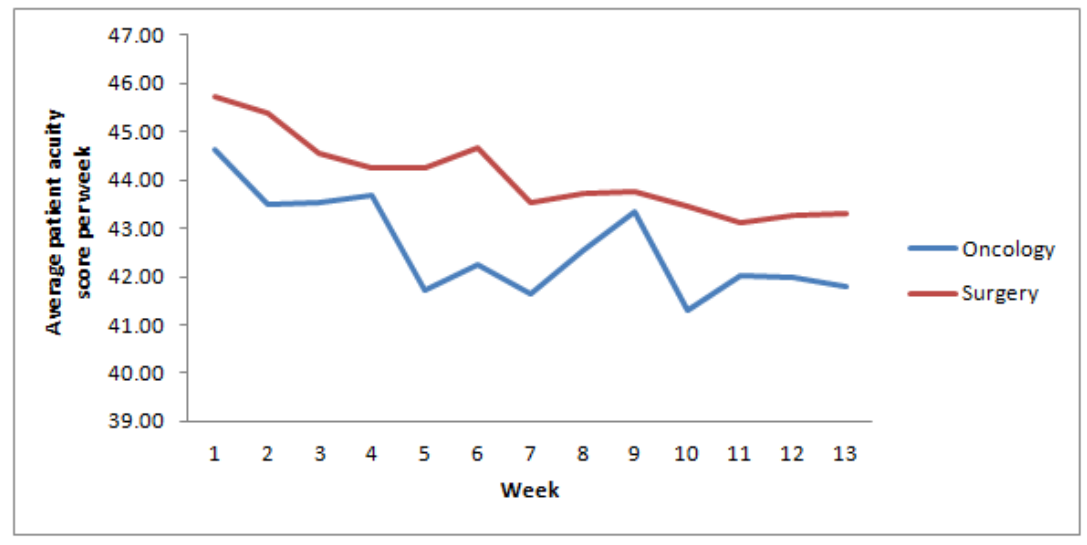

Figure 3.1: Trend of average patient acuity score based on the thirteen week period for both oncology and surgery nursing units

The average acuity scores of patients in the oncology and surgery units are 42.58 and 44.10, respectively. Figure 3.1 shows the trend of the average acuity score over time. As can seen, the average acuity scores change from week to week. Moreover, Figure 3.1 shows that there is a significant difference between patient characters for two nursing units. This is seen more clearly in Figure 3.2, which shows the percentage of patient who having a specific indicator. As shown in the figure, the frequency and distribution of patient acuity indicators are different for two nursing units. For instance, for indicator 5 (ADL Assistance: 2-3 caregivers), the percentage of oncology patients who have this indicator is $32.50 \%$ while $63.22 \%$ of surgery patients have the same indicator.

According to our data analysis patients with the same or close acuity scores can have very different patient acuity indicators. For example, two patients were admit- 


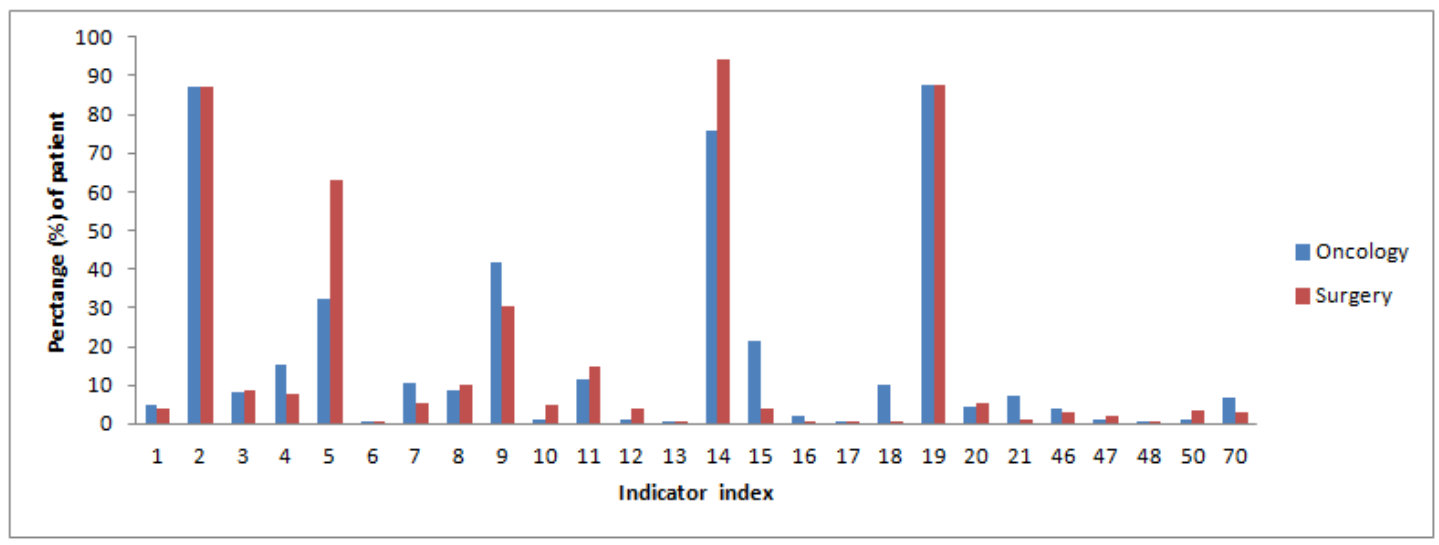

Figure 3.2: Percentage of patients who has a specific patient acuity indicator

ted to oncology unit of the hospital in the first day of January (see Table 3.1). Their patient type scores and complexity type scores are the same and the patient acuity scores are equal to 42. The first patient has indicator 2, 5, 14, and 19 while second one has indicator 2, 9, 14, and 19. Table 3.1 shows several similar examples, these examples clearly show that the scores do not guarantee the same patient acuity indicators distribution. Therefore, how much each nurse perceives the workload of a given patient can be different. Thus, we aim at focusing on individual nurses and how they perceive the workload of a given task and acuity level to assign nurses to patients.

Table 3.2 lists the available patient information recorded in the AcuityPlus PCS used at the University of Missouri Hospital. In addition to these data columns, the database also contains patient acuity indicator data. 


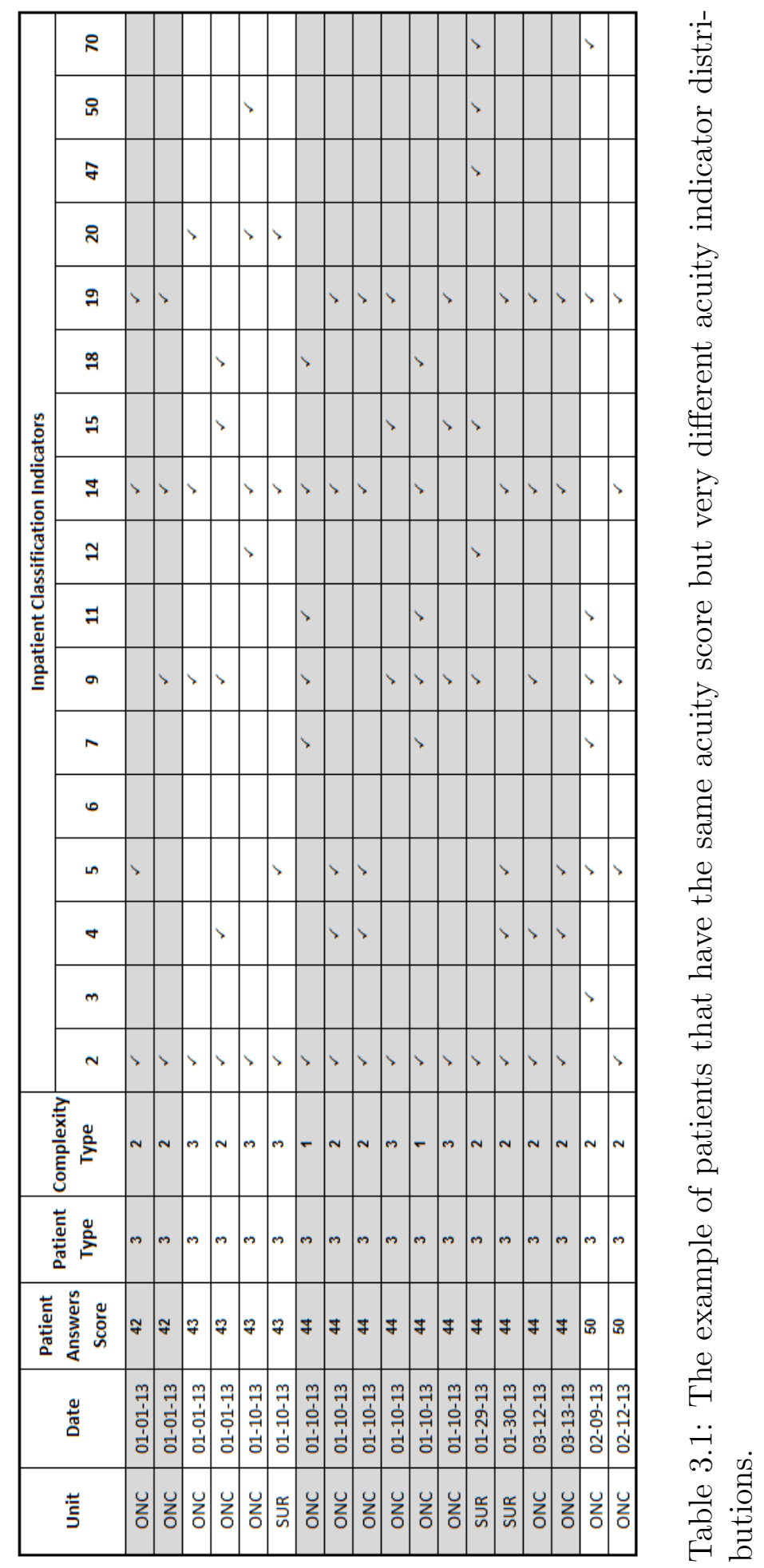


Table 3.2: Patient information recorded in the AcuityPlus database obtained from the University of Missouri Hospital.

\begin{tabular}{|l|}
\hline Unit \\
\hline Date \\
\hline Patient Answer Score \\
\hline Patient Type \\
\hline Complexity Type \\
\hline Room \\
\hline Arrival Time \\
\hline
\end{tabular}

\subsection{Nurse Workload Survey}

We conducted a survey with surgery and oncology nursing units at the University of Missouri Hospital to gather information to understand nurses' perceptions on the impact of various patient acuity indicators identified in the QuadraMed Acuity Plus system on nurses' workload. 45 nurses (25 from oncology nursing unit, 20 from surgery nursing unit) responded the survey.

The survey consists of seven questions (see Appendix B). The first five questions aim at identifying the characteristic of nurses, which include ages, gender, experience level, the highest degree, and the unit at which the nurse work. The central questions to the survey are intended to assess the impact of patient acuity indicators on nurses' workload. These questions are use a 6 points Likert scale, which forces respondents to choose non-neutral options. Chomeya (2010) compared Likert scale 6 and Likert scale 5 to determine which one is more reliable and discriminating and concluded that Likert scale 6 is more advantageous for using in questionnaires. Therefore, a 6 points Likert scale shown in Table 3.3 is used in our survey to assess the impact of patient acuity indicators on nurses' workload, in which weight 1 represents no impact 
on workload, while weight 6 shows extreme impact on workload.

Table 3.3: Likert scale used in nurse workload survey

\begin{tabular}{|l|l|}
\hline 1 & No Impact on Workload \\
\hline 2 & Slight Impact on Workload \\
\hline 3 & Some Impact on Workload \\
\hline 4 & Moderate Impact on Workload \\
\hline 5 & High Impact on Workload \\
\hline 6 & Extreme Impact on Workload \\
\hline
\end{tabular}

All respondents of survey are registered nurses. The experience distribution of nurses is given in Table 3.4, for instance. Furthermore,The average age of the nurses across two units is 38.67 years $(\mathrm{N}=45)$, and the significant majority of nurses $(80.56 \%)$ are female.

Figure 3.3 provides average scores for each patient acuity indicators. Table 3.5 mean, standard deviation, minimum, maximum score for each acuity indicator. As can be seen, perceived impact of patient acuity indicators on workload can be quite different for different nurses. Therefore, we develop an individualized survey-based workload model using patient acuity indicators. The survey-based workload model is described below.

Survey-Based Workload (SBW) Model: We develop a simple workload model for each individual nurse given their responses to the survey question, which asks the 
Table 3.4: The experience distribution of nurses

\begin{tabular}{|l|l|}
\hline Years & Percentage of Nurses \\
\hline $1-5$ & $66.67 \%$ \\
\hline $6-10$ & $4.44 \%$ \\
\hline $11-15$ & $6.67 \%$ \\
\hline $16-20$ & $2.22 \%$ \\
\hline $21-25$ & $15.56 \%$ \\
\hline $26-$ & $4.44 \%$ \\
\hline
\end{tabular}

impact of patient acuity indicators on their workload. Based on these responses, we calculate how much each the workload of a nurse increase when a patient is assigned by simply adding the scores from the survey for each indicator that the patient has. According to this workload model, the same patient might result in a very different workload profiles for different nurses. This is different from using acuity scores for assignment process because the acuity score of a patient is fixed regardless of which nurse cares for that patient.

Survey-based average perceived workload for each patient acuity indicator is almost identical between nurses at the oncology and surgery nursing units. As can be seen from Figure 3.4, many times the average survey-based perceived workload for the surgery unit is slightly higher than the oncology unit.

The standard deviation of each patient acuity indicators based on the nurse survey workload is provided in Figure 3.5. According to the figure, distribution of nurses' 


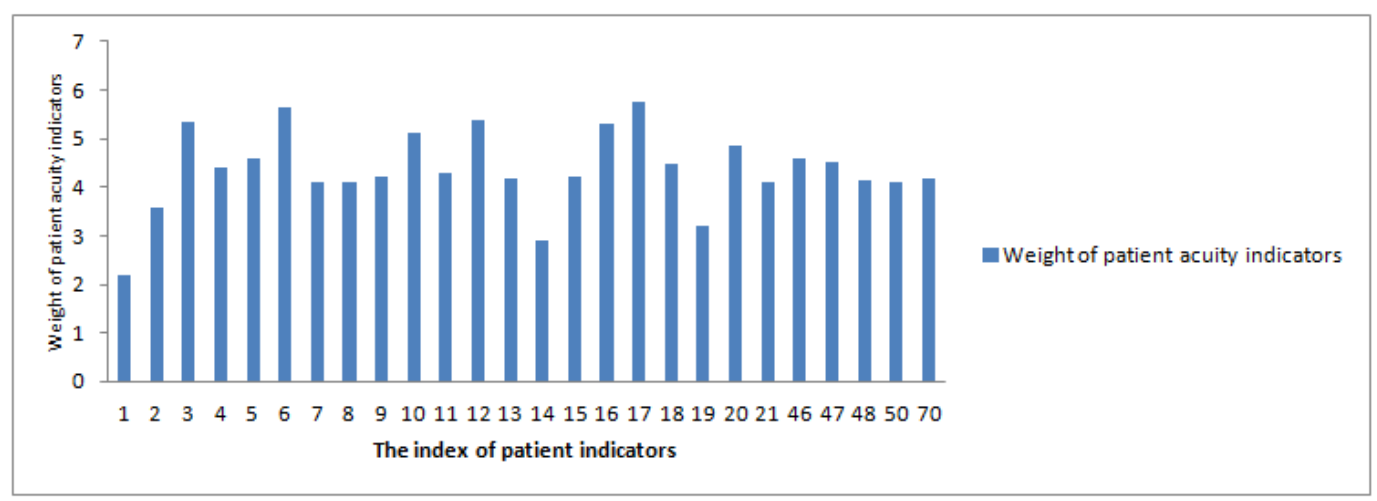

Figure 3.3: The average scores of patient acuity indicators based on the survey. The number on the $\mathrm{x}$-axis are the indices of the patient acuity indicators, the corresponding names of which are given in Table 2.3

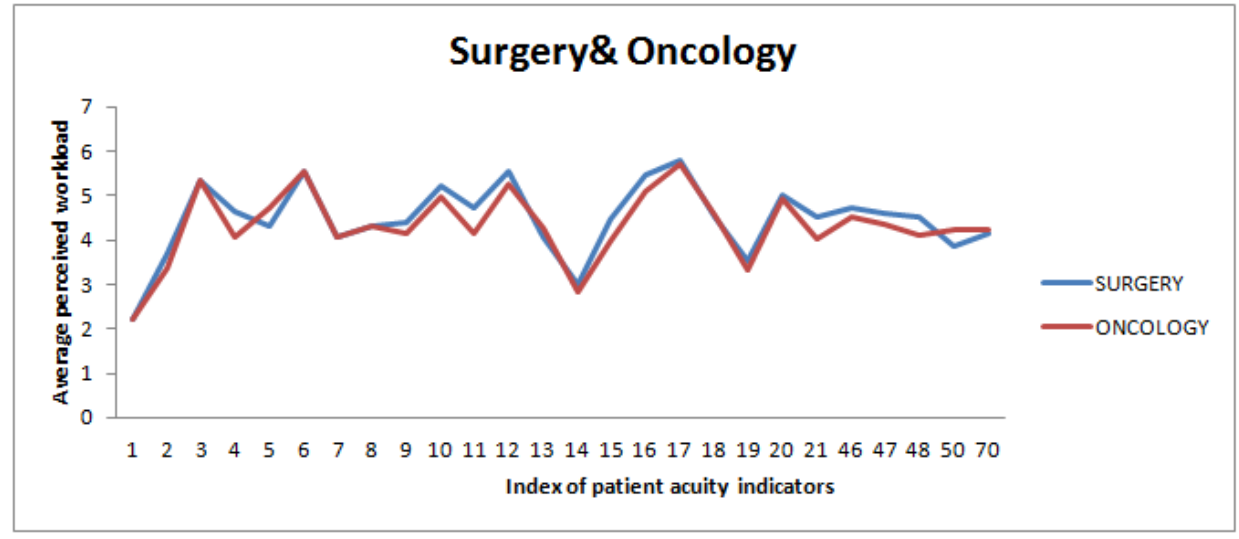

Figure 3.4: Survey-based average perceived workload of each patient acuity indicators for oncology and surgery nursing units. 
perceived workload between the oncology and surgery units for each patient acuity indicators differs from each other. This implies that using an individualized nurse workload model can help to make better assignment decisions.

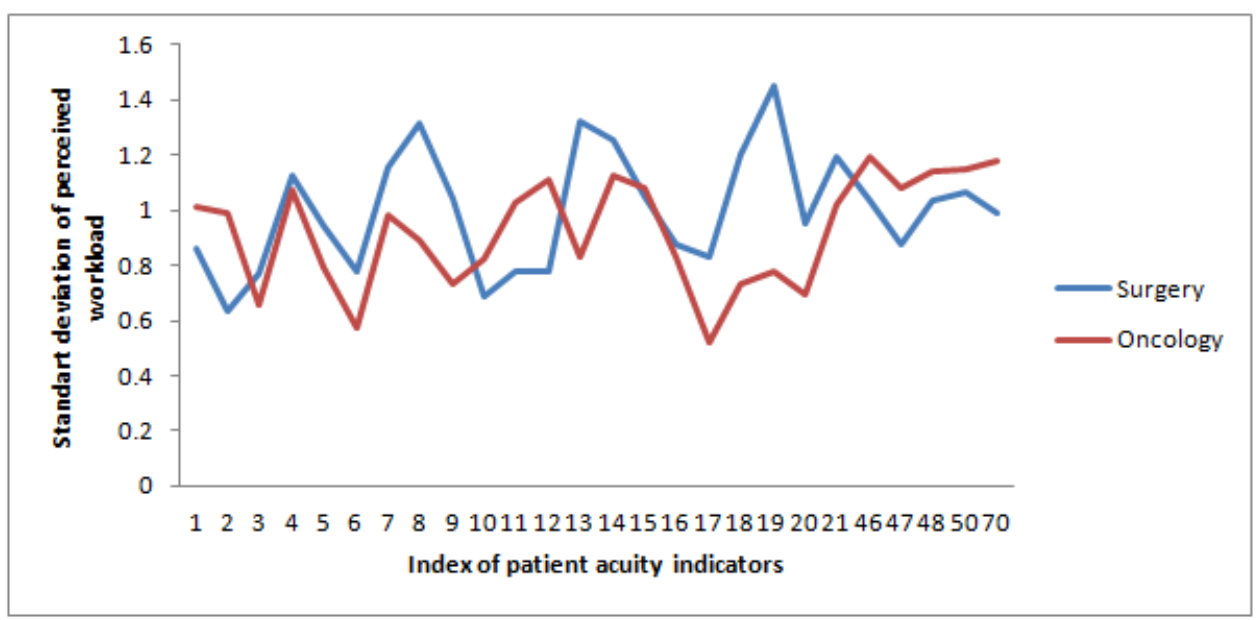

Figure 3.5: The standard deviation of survey-based average perceived workload of each patient acuity indicators for the oncology and surgery nursing units.

We have also performed analysis to determine the relationship between patient type from the AcuityPlus and survey-based nurse perceived workload. As seen in Figure 3.6. In addition, regression analysis show that the relationship between the patient type and survey-based nurse perceived workload is statistically significant for both units $P$-value $=0.001$ for the oncology nursing and $(P-$ value $=0.003$ for the surgery nursing unit). According to these results, the perceived workload is positively positively proportional with patient type.

The relationship between the complexity type of patient from the AcuityPlus and the survey-based perceived workload is shown in the Figure 3.7. Moreover, according to the regression analysis, the relationship between the complexity type and survey- 


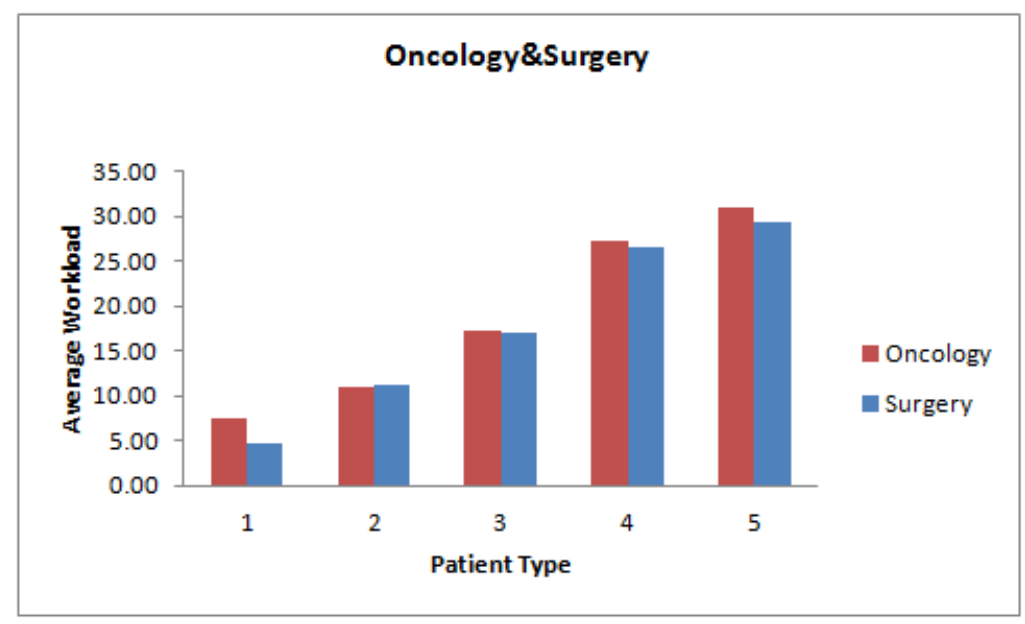

Figure 3.6: The relationship between the survey-based perceived workload and the patient type from AcuityPlus data.

based perceived workload for the oncology nursing unit $(P-$ value $=0.14>a=0.05)$ is not significant while for the surgery nursing unit $(P-$ value $=0.01<a=0.05)$ is significant for surgery unit. This indicates that complexity type may not accurately predict the perceived workload.

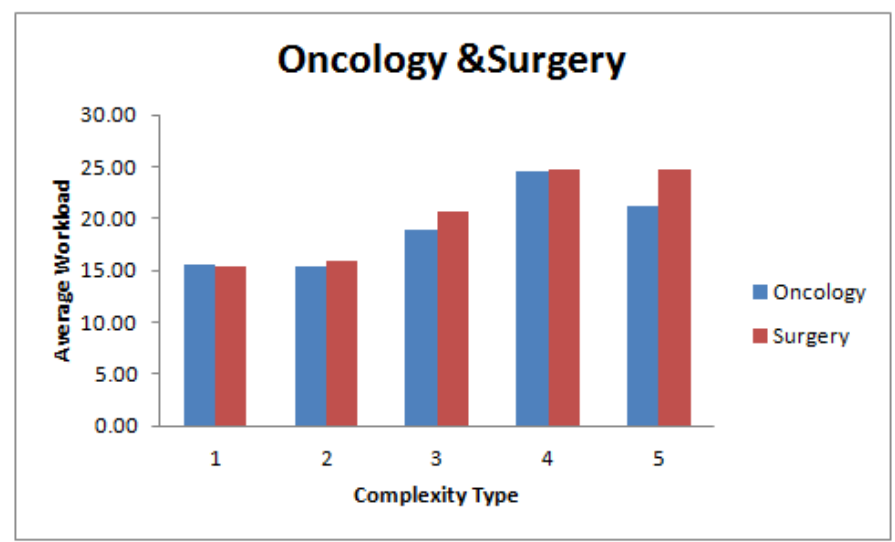

Figure 3.7: The relationship between the survey-based perceived workload and the complexity type of patient from the AcuityPlus data. 


\begin{tabular}{|c|c|c|c|c|c|}
\hline$R$ & $\underset{\sim}{\tilde{\gamma}}$ & $\stackrel{ }{7}$ & $\stackrel{8}{i}$ & $\underset{+}{\stackrel{-}{~}}$ & : \\
\hline in & $\underset{\forall}{J}$ & $\stackrel{m}{]}$ & 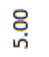 & & : \\
\hline gg & $\stackrel{g}{\gamma}$ & & $\stackrel{8}{i}$ & ه্ণ & : \\
\hline f & $\stackrel{f}{f}$ & & $\stackrel{8}{\circ}$ & ه্ণ & : \\
\hline$q$ & $\begin{array}{l}\overrightarrow{+} \\
\dot{+}\end{array}$ & $\stackrel{\overbrace{}}{\rightarrow}$ & 帘 & $\underset{+}{8}$ & : \\
\hline$\pi$ & 年 & 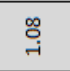 & $\begin{array}{l}8 \\
\\
\end{array}$ & 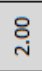 & : \\
\hline ㅇ. & 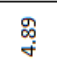 & $\begin{array}{l}\stackrel{\infty}{0} \\
\stackrel{0}{0}\end{array}$ & 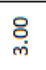 & 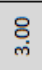 & : \\
\hline 9 & 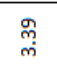 & 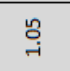 & 学 & $\underset{+}{8}$ & 8 \\
\hline$\stackrel{\sim}{\prime \prime}$ & 号 & गु่ & $\begin{array}{l}8 \\
\dot{+}\end{array}$ & $\stackrel{8}{8}$ & 8 \\
\hline A & $\begin{array}{l}\infty \\
\stackrel{\infty}{n} \\
\dot{n}\end{array}$ & d: & $\underset{\dot{m}}{\stackrel{8}{ }}$ & $\underset{m}{8}$ & $\stackrel{8}{\circ}$ \\
\hline$\stackrel{7}{\prime}$ & 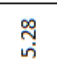 & $\begin{array}{c}\ddot{0} \\
0 \\
0\end{array}$ & 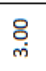 & $\underset{m}{8}$ & 8 \\
\hline ص & 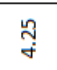 & 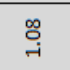 & $\begin{array}{l}8 \\
\dot{g} \\
\end{array}$ & $\stackrel{8}{\text { i }}$ & 8 \\
\hline$\exists$ & গิ & $\underset{7}{7}$ & $\begin{array}{l}8 \\
\dot{+}\end{array}$ & $\underset{+}{8}$ & i̊ \\
\hline 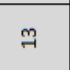 & 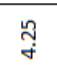 & ઼ָ & $\begin{array}{l}8 \\
\dot{j}\end{array}$ & $\stackrel{8}{8}$ & 8 \\
\hline$\approx$ & $\begin{array}{l}\text { ơ } \\
\text { in }\end{array}$ & ুू & $\stackrel{8}{\text { or }}$ & $\underset{+}{\stackrel{-}{8}}$ & 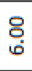 \\
\hline$\Rightarrow$ & $\vec{p}$ & ฏू & $\begin{array}{l}8 \\
\\
\end{array}$ & $\underset{\text { i }}{8}$ & 8 \\
\hline 9 & $\begin{array}{l}0 \\
\text { og } \\
\text { in }\end{array}$ & $\begin{array}{l}\text { R } \\
\text { o }\end{array}$ & 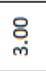 & 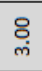 & : \\
\hline$a$ & 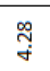 & $\stackrel{\ddot{0}}{0}$ & $\underset{+}{8}$ & $\stackrel{\text { i }}{\text { de }}$ & $\stackrel{8}{8}$ \\
\hline$\infty$ & 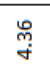 & 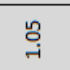 & ষ্ণ & $\stackrel{\text { i }}{\text { de }}$ & $\stackrel{0}{\circ}$ \\
\hline N & $\overrightarrow{7}$ & ț & $\begin{array}{l}8 \\
\dot{q} \\
\end{array}$ & $\underset{\text { i }}{8}$ & : \\
\hline 0 & $\begin{array}{l}\overrightarrow{0} \\
\dot{\omega}\end{array}$ & 㕝 & $\stackrel{\text { i }}{8}$ & $\begin{array}{l}\stackrel{8}{\circ} \\
+\end{array}$ & : \\
\hline in & 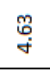 & 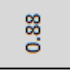 & $\underset{\sim}{\stackrel{\text { }}{ }}$ & $\underset{\sim}{\stackrel{\leftrightarrow}{\circ}}$ & 8 \\
\hline ナ & $\vec{\sigma}$ & ğ & 帘 & $\underset{-}{\stackrel{-}{~}}$ & 8 \\
\hline$m$ & 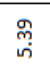 & 。̊ & 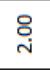 & $\underset{+}{8}$ & 8 \\
\hline$N$ & 总 & $\begin{array}{c}\substack{\infty \\
0 \\
0} \\
\end{array}$ & $\underset{i}{8}$ & $\stackrel{\text { i }}{\text {. }}$ & 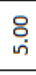 \\
\hline 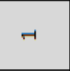 & $\stackrel{9}{\mathrm{i}}$ & ณू & $\underset{+}{+}$ & $\underset{+}{\stackrel{+}{0}}$ & 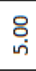 \\
\hline 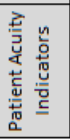 & $\begin{array}{l}\text { [D } \\
\stackrel{\mathbb{W}}{\Sigma}\end{array}$ & 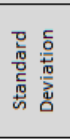 & 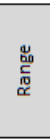 & 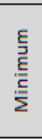 & 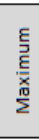 \\
\hline
\end{tabular}




\section{Chapter 4}

\section{Nurse-Patient Assignment Problem}

One of the studies that are closely related to ours was done by (Mullinax et al. 2002). They focused on a neonatal intensive care unit for assigning a group of infants to staff nurses. In their study, they developed an integer linear program to balance workload of nurses. Since the intensive care unit they studied within their research did not have a patient acuity system, as a first step, the authors developed a patient acuity system to quantify patient care needs. Next, using the patient acuity points, they developed a model which assigns infants to staff nurses so that the total acuity score assigned to nurses as close to each other as possible.

Similar to Mullinax's model, the nurse patient assignment policy at the University of Missouri Hospital is the based on the patient acuity scores. Therefore, to mimic this policy, we adopted Mullinax's integer linear programming model that using patient acuity scores from the AcuityPlus PCS. As discussed in Section 3.2, how a nurse 
perceives the impact of a patient acuity indicator on their workload is subjective, we developed three additional assignment models using the survey-based workload model described in Section 3.2. The detail of each model is described below. These new models are motivated by our belief that balancing the workload based on the perception of individual nurse will result in better nurse satisfaction and therefore increase the retention rate of nurses.

\subsection{Nurse-Patient Assignment Model with an Ob- jective of Balancing Patient Acuity Scores from AcuityPlus PCS}

In this section, we adopt Mullinax's nurse-patient assignment model with an objective of balancing patient acuity scores from the AcuityPlus PCS. This model will be used as a baseline which represents current state of practice. We first define notation, which is followed by the formulation.

\section{Sets and Indices}

- $\mathcal{N}$ is the set of all nurses, indexed by $n$.

- $\mathcal{P}$ is the set of all patients, indexed by $p$.

- $\mathcal{K}$ is the set of patients' acuity indicators, indexed by $k$.

\section{Parameters}

- $l$ and $u$ are the minimum and maximum number of patients that can be assigned to a nurse, respectively. 
- $a_{p}$ is the acuity score of a patient $p$.

\section{Decision Variables}

- $x_{n p}= \begin{cases}1, & \text { if patient } p \text { is assigned to nurse } n \\ 0, & \text { otherwise. }\end{cases}$

- $z^{\max }$ is the maximum total patient acuity score assigned to a nurse.

- $z^{\text {min }}$ is the minimum total patient acuity score assigned to a nurse.

\section{Objective Function}

$$
\min z^{\max }-z^{\min }
$$

The objective function ensures that the difference between the worst-off and bestoff nurse (in terms of the total acuity scores assigned to them) is minimized. This results in the most equitable (i.e., balanced) assignment in terms of distribution of acuity scores among nurses.

\section{Constraints}

$$
\begin{aligned}
& \sum_{n \in \mathcal{N}} x_{n p}=1, \quad \forall p \in \mathcal{P} \\
& \sum_{p \in \mathcal{P}} x_{n p} \geq l, \quad \forall n \in \mathcal{N} \\
& \sum_{p \in \mathcal{P}} x_{n p} \leq u, \quad \forall n \in \mathcal{N} \\
& \sum_{p \in \mathcal{P}} a_{p} x_{n p} \leq z^{\max }, \quad \forall n \in \mathcal{N} \\
& \sum_{p \in \mathcal{P}} a_{p} x_{n p} \geq z^{\min }, \quad \forall n \in \mathcal{N}
\end{aligned}
$$


The constraint set (4.2) assures that each patient is assigned to exactly one nurse. The constraint sets (4.3) and (4.4) assure that each nurse is assigned to at least $l$ and at most $u$ patients. The constraints sets (4.5) and (4.6) define variables $z^{\max }$ and $z^{\text {min }}$, respectively.

\subsection{Nurse-Patient Assignment Model Based on Bal- ancing Survey-Based Workload}

In this section, we formulate nurse-patient assignment model by incorporating how much nurse perceives their workload. This model only tries to minimize range between maximum and minimum assigned acuity score. That is the reason, in the next section, we introduce two new model to help us to both balance and decrease nurses' workload.

\section{Parameters}

- $a_{p k}= \begin{cases}1, & \text { if patient } p \text { is classified as having patient acuity indicator } k \\ 0, & \text { otherwise }\end{cases}$

- $w_{n k}$ is survey-based workload score for nurse $n$ and indicator $k$.

\section{Decision Variables}

- $x_{n p}= \begin{cases}1, & \text { if the patient } \mathrm{p} \text { is assigned to nurse } \mathrm{n}, \\ 0, & \text { otherwise }\end{cases}$

- $z^{\max }$ is the maximum total survey-based workload score assigned to a nurse

- $z^{\text {min }}$ is the minimum total survey-based workload score assigned to a nurse 


\section{Objective Function}

$$
\min z^{\max }-z^{\min }
$$

The objective function tries to decrease range between the assigned maximum and minimum patient acuity scores, thus balancing nurse workload, respectively.

\section{Constraints}

Constraint Set (4.2)

Constraint Set (4.3)

Constraint Set (4.4)

$$
\begin{aligned}
& \sum_{p \in \mathcal{P}} \sum_{k \in \mathcal{K}} w_{n k} a_{p k} x_{n p} \leq z^{\max }, \quad \forall n \in \mathcal{N} \\
& \sum_{p \in \mathcal{P}} \sum_{k \in \mathcal{K}} w_{n k} a_{p k} x_{n p} \leq z^{m i n}, \quad \forall n \in \mathcal{N}
\end{aligned}
$$

The constraint set (4.2), (4.3) and (4.4) are same as mentioned in the previous section. Constraint (4.8) and (4.9) sets up $z^{\max }$ and $z_{k}^{\min }$ assigned weighted patient acuity indicators among all nurses, respectively. 


\subsection{Nurse-Patient Assignment Model Based on Minimizing Total Survey-Based Workload}

As mentioned in the previous section, minimizing range between the maximum and minimum assigned patient acuity scores is not only adequate to balance nurse workload. Therefore, minimizing total acuity score objective will be considered in this section in order to decrease the workload.

\section{Parameters}

- $a_{p k}= \begin{cases}1 \quad \text { if the patient } p \text { is classified as having patient acuity indicator } k \\ 0 \quad \text { otherwise }\end{cases}$

- $w_{n k}$ is survey-based workload score for nurse $n$ and indicator $k$.

\section{Decision Variables}

- $x_{n p}= \begin{cases}1 & \text { if the patient } \mathrm{p} \text { is assigned to nurse } \mathrm{n}, \\ 0 & \text { otherwise }\end{cases}$

- $z_{\text {total }}$ is the total survey-based acuity score assigned all nurses

\section{Objective Function}

$$
\min z_{\text {total }}
$$

The objective function in this section provides us to reduce balancing workload to a reasonable level. $z_{\text {total }}$ is the assigned survey-based acuity score to all nurses that is calculated based on the how much nurses perceive the patient acuity indicators. 


\section{Constraints}

Constraint Set (4.2)

Constraint Set (4.3)

Constraint Set (4.4)

$$
\sum_{n \in \mathcal{N}} \sum_{p \in \mathcal{P}} \sum_{k \in \mathcal{K}} w_{n k} a_{p k} x_{n p}=z_{\text {total }},
$$

The constraint set 4.2, 4.3 and 4.4 are same as mentioned in the previous section. The constraint (4.11) calculates the total patient acuity score assigned to all nurse, which includes survey-based nurses' preference about patient acuity indicators.

\subsection{Nurse-Patient Assignment Model Based on Minimizing Maximum Survey-Based Workload}

The model formulations in this section help us to decrease maximum assigned patient acuity scores to nurses.

\section{Parameters}

- $a_{p k}= \begin{cases}1 & \text { if the patient } p \text { is classified as having patient acuity indicator } k \\ 0 & \text { otherwise }\end{cases}$

- $w_{n k}$ is survey-based workload score for nurse $n$ and indicator $k$.

\section{Decision Variables}

- $x_{n p}= \begin{cases}1 & \text { if the patient } \mathrm{p} \text { is assigned to nurse } \mathrm{n}, \\ 0 & \text { otherwise }\end{cases}$ 
- $z_{\max }$ is the maximum survey-based acuity score among nurses

\section{Objective Function}

$$
\min z_{\max }
$$

The objective function considers minimizing the maximum survey-based acuity score assigned all nurse.

\section{Constraints}

Constraint Set (4.2)

Constraint Set (4.3)

Constraint Set (4.4)

$$
\sum_{p \in \mathcal{P}} \sum_{k \in \mathcal{K}} w_{n k} a_{p k} x_{n p} \leq z_{\text {max }}, \quad \forall n \in \mathcal{N}
$$

The constraint set (4.2), (4.3) and (4.4) are same as mentioned in the Section 4.1. The constraint (4.13) arrange maximum total survey-based acuity scores assigned to a nurse. 


\section{Chapter 5}

\section{Numerical Results}

As mentioned in previous chapters, we used survey data and data from AcuityPlus PCS obtained from the oncology and surgery nursing units at the university of Missouri. In this chapter, we present numerical results based on these real data to show that the nurse-patient assignment models developed in Chapter 4 effectively balance and reduce the perceived workload.

We run 200 random experiments (100 for the oncology unit and 100 for the surgery unit to show the effectiveness of the nurse-patient assignment model described in Chapter 4 in terms of balancing and decreasing the perceived workload. In the each experiments, 30 patients are randomly selected from the 2865 and 3241 patients from oncology and surgery nursing, respectively. The number of patients used in the experiments are close to the average number of admitted patient per day for aforementioned nursing. Moreover, in each experiment 5 nurses are randomly selected among 36 nurses, who have participated in the survey from the oncology and surgery nursing units. 
The nurse-patient assignment models described in Chapter 4 were implemented using Python (version 2.7.3) (Python Software Foundation 2013) (see Appendix C) and solved by the Gurobi solver (Gurobi Optimization 2013). Running time for each experiment takes only a few minutes on a personal computer with an Intel core i5 $(2.30 \mathrm{GHz})$ processor and $4 \mathrm{~GB}$ RAM.

Table 5.1: Notations used in the remaining chapters for models described in Chapter 4

\begin{tabular}{|l|l|l|}
\hline Model & Notation & Description \\
\hline Model 1 & M1 & $\begin{array}{l}\text { Nurse-Patient Assignment Model } \\
\text { with an Objective of Balancing } \\
\text { Patient Acuity Scores from Acu- } \\
\text { ityPlus PCS }\end{array}$ \\
\hline Model 2 & M2 & $\begin{array}{l}\text { Nurse-Patient Assignment Model } \\
\text { Based on Balancing Survey- } \\
\text { Based Workload }\end{array}$ \\
\hline Model 3 & M3 & $\begin{array}{l}\text { Nurse-Patient Assignment Model } \\
\text { Based on Minimizing Total } \\
\text { Survey-Based Workload }\end{array}$ \\
\hline Model 4 & M4 & $\begin{array}{l}\text { Nurse-Patient Assignment Model } \\
\text { Based on Minimizing Maximum } \\
\text { Survey-BasedWorkload }\end{array}$ \\
\hline
\end{tabular}

We compared the performance of various assignment models described in Chapter 4 by applying ANOVA (Minitab Inc. 2009) to the mean and standard deviation of perceived workload among nurses for each nurse-patient assignment model. The result of ANOVA comparing the mean perceived workload for the oncology and surgery units 
are given in Table 5.2. In both tables (a) and (b), Pvalue $<0.05$, which implies that statistically at least the mean of the models is different. We used Fisher's Least Significant Difference (LSD) to understand which pair of means are different as given in Table 5.3. According to LSD, models 1 and 2 and model 3 and model 4 form a group, which implies that the difference in the mean perceived workload for model 1 and 2 and model 3 and model 4 is not statistically significant for the oncology unit (Table 5.3(a)) LSD results in three groups for the surgery unit (Table 5.3(b))

Table 5.2: ANOVA results comparing the mean perceived workload of the four nursepatient assignment model described in Chapter 4

(a) Oncology unit

\begin{tabular}{lllllll} 
ANOVA & \multicolumn{1}{l}{} \\
\hline Source of Variation & SS & df & MS & F & P-value & F crit \\
\hline Between Groups & 4301.37 & 3.00 & 1433.79 & 27.58 & 0.00 & 2.63 \\
Within Groups & 20585.03 & 396.00 & 51.98 & & & \\
Total & 24886.40 & 399.00 & & & & \\
\hline
\end{tabular}

(b) Surgery unit

\begin{tabular}{lllllll} 
ANOVA & \multicolumn{1}{l}{} \\
\hline Source of Variation & SS & df & MS & F & P-value & F crit \\
\hline Between Groups & 3806.31 & 3.00 & 1268.77 & 25.41 & 0.00 & 2.63 \\
Within Groups & 19771.47 & 396.00 & 49.93 & & & \\
Total & 23577.78 & 399.00 & & & & \\
\hline
\end{tabular}

Table 5.3: Result of the LSD method comparing the mean perceived workload for the four assignment models. The difference in the mean perceived workload for the models in the same group is not statistically significant.

(a) Oncology unit

\begin{tabular}{lllc}
\hline Model & N & Mean & Grouping \\
\hline M1 & 100 & 84.545 & A \\
M2 & 100 & 82.87 & A \\
M3 & 100 & 76.46 & B \\
M4 & 100 & 78.327 & B \\
\hline \multicolumn{4}{l}{ Grouping Information Using Fisher Method }
\end{tabular}

(b) Surgery unit

\begin{tabular}{lllc}
\hline Model & N & Mean & Grouping \\
\hline M1 & 100 & 85.63 & A \\
M2 & 100 & 82.777 & B \\
M3 & 100 & 79.398 & C \\
M4 & 100 & 77.622 & C \\
\multicolumn{4}{l}{ Grouping Information Using Fisher Method }
\end{tabular}


The differences between the mean perceived workload of the four assignment models can be also clearly seen in Figure 5.1 and 5.2. For the both units, Model 1 has the largest perceived workload values and while Model 3 has the smallest perceived workload values. According to these results, using an objective of balancing acuity scores(Model 1)or perceived workload(Model 2) may not be sufficient to reduce overall perceived workload, an objective of minimizing total (Model 3) or maximum (Model 4) must be used. While reducing overall perceived workload is important, an equally (if not more) important criterion is the balance between the workload distributed among nurses.

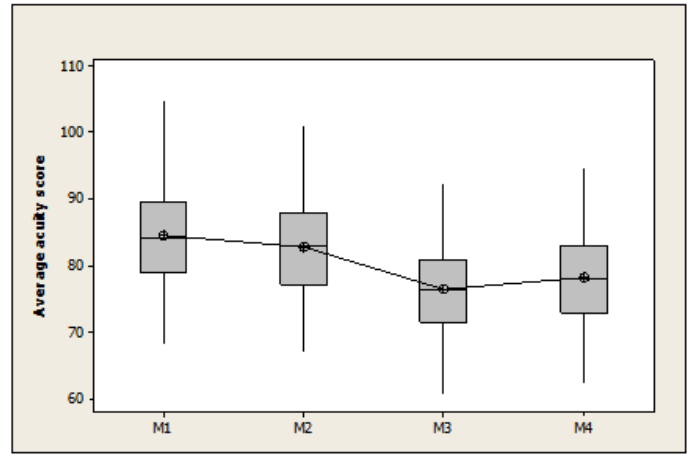

(a) Oncology unit

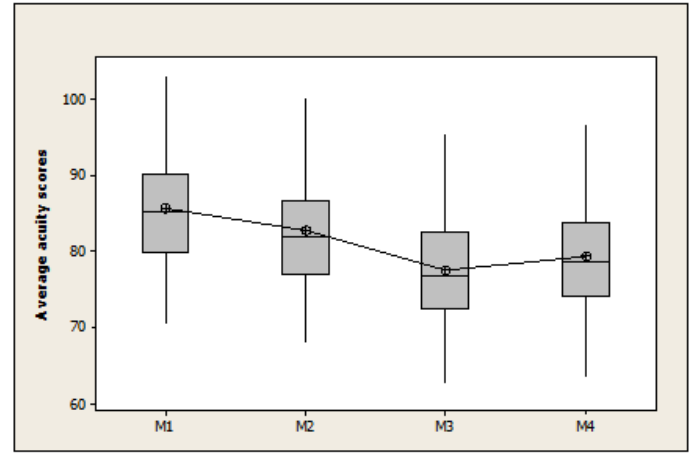

(b) Surgery unit

Figure 5.1: Boxplot comparing the mean perceived workload for different models

Comparing four assignment model's standard deviation is also significant in terms of showing that our new models are robust and evenly balance workload of nurses. As seen in the Table 5.4, difference between the standard deviation of models are statistically significant. Moreover, in order to compere pair, LSD method is used to show whether they are grouped together or not as shown in Table 5.5.

Figure 5.3 demonstrates that the largest mean, and value of standard deviation. The standard deviation for Model 2 is zero, since this model perfectly balance the 


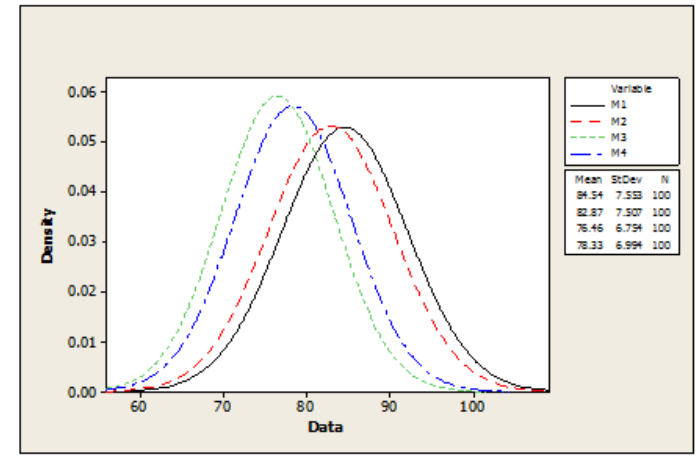

(a) Oncology unit

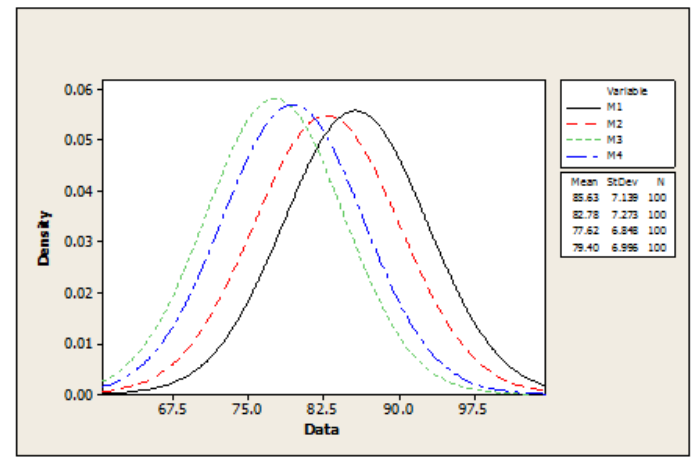

(b) Surgery unit

Figure 5.2: Distribution of the mean perceived workload for different models

Table 5.4: ANOVA results comparing the standard deviation perceived workload of the four nurse-patient assignment model described in Chapter 4 .

(a) Oncology unit

ANOVA

\begin{tabular}{lllllll}
\hline Source of Variation & SS & df & MS & F & P-value & F crit \\
\hline Between Groups & 33390.96 & 3.00 & 11130.32 & 814.91 & 0.00 & 2.63 \\
Within Groups & 5408.68 & 396.00 & 13.66 & & & \\
& & & & & & \\
Total & 38799.64 & 399.00 & & & & \\
\hline
\end{tabular}

(b) Surgery unit

ANOVA

\begin{tabular}{lllllll}
\hline Source of Variation & SS & df & MS & F & P-value & F crit \\
\hline Between Groups & 26185.39 & 3.00 & 8728.46 & 737.84 & 0.00 & 2.63 \\
Within Groups & 4684.58 & 396.00 & 11.83 & & & \\
& & & & & & \\
Total & 30869.97 & 399.00 & & & & \\
\hline
\end{tabular}


Table 5.5: Result of the LSD method comparing the standard deviation perceived workload for the four assignment models. The difference in the standard deviation perceived workload for the models in the same group is not statistically significant.

(a) Oncology unit

\begin{tabular}{lllc}
\hline Model & $\mathrm{N}$ & Mean & Grouping \\
\hline M1 & 100 & 14.903 & $\mathrm{~B}$ \\
M2 & 100 & 0 & $\mathrm{C}$ \\
M3 & 100 & 21.135 & $\mathrm{~A}$ \\
M4 & 100 & 0.58 & $\mathrm{C}$ \\
\hline \multicolumn{4}{l}{ Grouping Information Using Fisher Method }
\end{tabular}

(b) Surgery unit

\begin{tabular}{lllc}
\hline Model & $\mathrm{N}$ & Mean & Grouping \\
\hline M1 & 100 & 17.269 & $\mathrm{~A}$ \\
M2 & 100 & 0.007 & $\mathrm{C}$ \\
M3 & 100 & 15.749 & $\mathrm{~B}$ \\
M4 & 100 & 0.735 & $\mathrm{C}$ \\
\hline \multicolumn{4}{l}{ Grouping Information Using Fisher Method }
\end{tabular}

workload of model. However, this model can not decrease balanced workload to desired level, which is the drawback of this model.

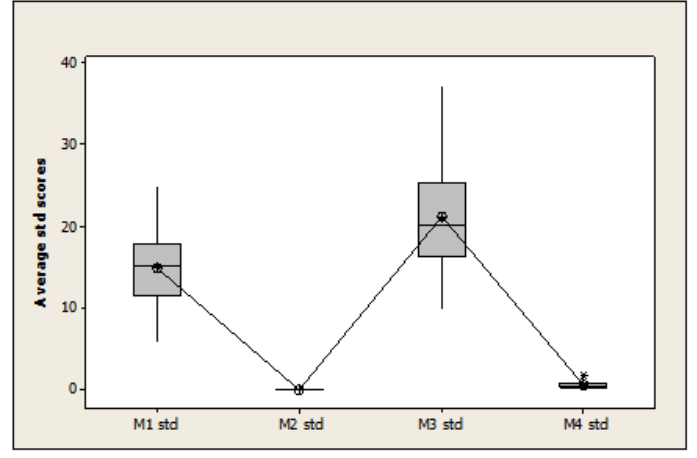

(a) Oncology unit

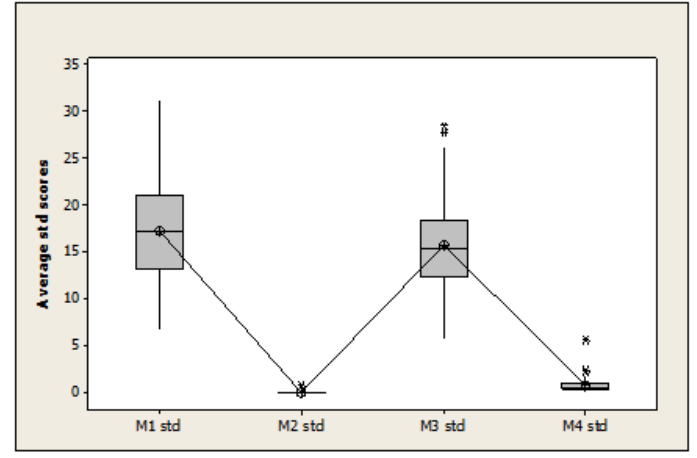

(b) Surgery unit

Figure 5.3: Boxplot comparing the standard deviation perceived workload for different models

The Figure 5.4 also provides detailed information about standard deviation perceived workload for four nurse patient assignment models both for the oncology and surgery nursing units. 


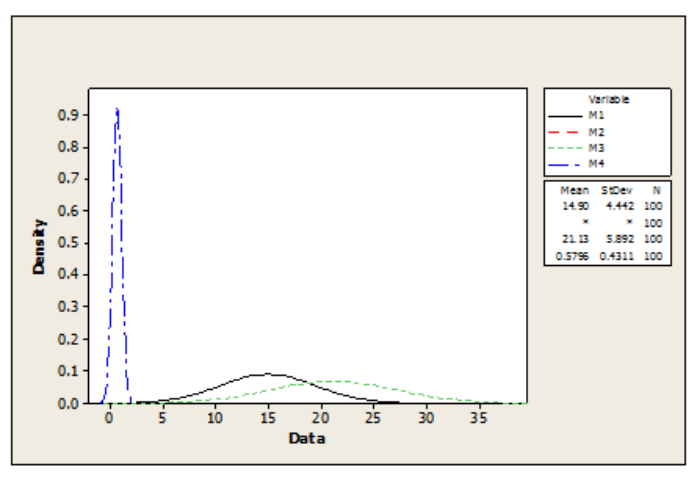

(a) Oncology unit

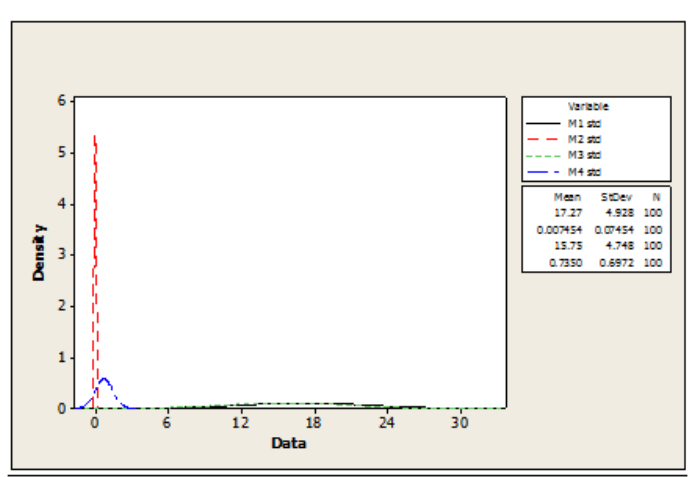

(b) Surgery unit

Figure 5.4: Distribution of the standard deviation perceived workload for different models

\subsection{Discussion}

Numerical results indicate that the nurse-patient assignments from model 2,3, and 4 are significantly better than Model 1, which is adopted from Mullinax et al. (2002), when evaluated using the survey-based perceived workload model described in Section 3.2. On the other hand, since patient acuity indicators given in the survey does not necessarily reflect the task or sub-task of nurses, more detailed models including nursing tasks or sub-task must be developed in order to accurately represent nurses workload. For example,link analysis can provide important information about the motions of nurses between points in the nursing units, since nurse workload does not only include caring for patients. Therefore, besides survey-based workload used in this thesis, quantified link analysis results must be incorporated into workload calculations. The cognitive pathway method can also be quantified to determine the impact of cognitive shifts and thereby incorporated into workload calculation.

Our models can be used in any hospital that has a patient classification system. Besides the patient classification systems requirement, nurses need to be surveyed 
several times to obtain a robust survey-based nurse workload model. 


\section{Chapter 6}

\section{Conclusion and Future Works}

In this thesis, we developed several nurse-patient assignment (NPA) models to balance and reduce nurses' perceived workload based on a survey. The first NPA model is adopted from Mullinax et al. (2002), which only minimizes the range between the maximum and minimum patient acuity scores assigned to all nurses. Since this model does not include how much nurses perceive a particular patient acuity indicators increase their workload, we incorporate a survey-based perceived workload model into Model 2. We also developed Model 3 and 4 to decrease the survey-based perceived workload of nurses. According to ANOVA analysis, these models significantly decreased the perceived workload of nurses. Therefore, these models can help improving nurses' work condition and retain them in nursing.

Model 2 efficiently balances the perceived workload while model 3 and 4 only reduce the perceived workload. Since both objectives of balancing and reducing perceived workload are very important, in future research, a weighted multi-objective function can be developed. Moreover, since this study just includes the surgery and 
oncology nursing units of an academic hospital, future research will focus on all units in an hospital to assess the broader applicability of our models.

In the survey, we have asked nurses about other factors that impact their workload. About $50 \%$ percent of nurses strikingly responded this survey question, and the given responses include detailed information about patient caring tasks. For this reason, a study can be performed for different units to determine other significant factors affecting workload and then improve the survey-based workload model used in this thesis.

Lastly, since patient classification system is implemented across all units in the hospital using the same patient indicators, a research could be conducted for analyzing whether the same indicator is incorporated the same way in different nursing units. 
Appendix A

Health Sciences Institutional Review Board Approval Letter 
February 8, 2013

This project was reviewed and approved by the University of Missouri - Columbia Health Sciences Institutional Review Board (HSIRB) according to the terms and conditions described below:

Project Number

Project Title

Principle Investigator

Primary Contact

Approval Date

Expiration Date

Approval Category

Level of Review

All data collected must exist on the shelf at

Exempt
1207004

Identifying relationships between patient acuity and types and frequencies of nursing activities

Sir, Mustafa Yasar

Sir, Mustafa Yasar

Feb 07, 2013

Feb 07, 2014

Exempt 45 CFR 46.101(b) $<4>$

All documents reviewed and approved can be found in digital documents and are highlighted green.

You are expected to comply with the requirements outlined in the MU HSIRB Policies

(http://research.missouriedu/hsirb/policies.htm). This includes reporting any unanticipated problems involving risk to research participants or others.

Changes in the conduct of the study, including consent process or materials, require submission of an amendment form which must be approved by the HSIRB prior to implementation of the changes. Changes in the source of study funding must also be reported.

According to federal regulations, this project requires IRB continuing review. As such, prior to the expiration date above, you must submit either an Exempt Annual Update (EAU) or the Completion/Withdrawal Form. If you have questions or require additional information, please contact us at (573) 882-3181 or irb@missouri.edu

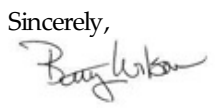

Betty Wilson

Compliance Officer, HS IRB 
Amendment for conducting a survey. This approval was added after first one. Project Number: 1207004

Review Number: 114747

Project Title: Identifying relationships between patient acuity and types and frequencies of nursing activities

Principal Investigator(s): Sir, Mustafa Yasar

Primary Contact: Sir, Mustafa Yasar

Approval Date: May 31, 2013

Your amendment above has been approved. Please log into the eIRB to access any approved documents. 
Appendix B

Survey 


\section{Nurse Workload Study<br>}

This survey is being conducted by researchers in the Department of Industrial and Manufacturing System Engineering The purpose of this survey is to gather information to understand nurses' perceptions on the impact of various patient acuity indicators identified in the QuadMed Acuity Plus system to classify patients on nurses' workload.

Participating in this survey is completely voluntary. All of your responses are completely anonymous and will be viewed only by the research team. The entire survey should require approximately 5-10 minutes to complete.

For the sake of the study, please give honest responses. Thank you in advance for participating. We appreciate your feedback and hope to return to the nursing units participating in this study with suggestions for balancing workload. Please contact Dr. Mustafa Y. Sir sirm@missouri.edu with any concerns or additional comments.

\section{What is your age?}

Age:

\section{How many years of experience do you have working as a nurse?}

( $1-5$

( $16-20$

6-10

( $21-25$

(11-15

Other (please specify)

\section{What is your gender?}

Female

Male

Transgender

\section{What is your highest nursing degree?}

Certified Nursing Assistant (CNA)

Licensed Practical Nurse (LPN)

Registered Nurse (RN)

Advanced Practical Registered Nurse (APRN)

Nurse Practitioner

Doctor of Nursing Practice (DNP)

\section{Which department do you work for?}

S Surgery

O Oncology 


\section{Nurse Workload Study<br>}

The following questions are related to patient acuity indicators that are taken from AcuityPlus System. Please mark your response on the scale next to each indicator.

\section{Please rate the following patient acuity indicators on their impact on your workload.}

\begin{tabular}{|c|c|c|c|c|c|c|}
\hline & $\begin{array}{l}\text { No Impact on } \\
\text { Workload }\end{array}$ & $\begin{array}{l}\text { Slight Impact } \\
\text { on Workload }\end{array}$ & $\begin{array}{l}\text { Some Impact } \\
\text { on Workload }\end{array}$ & $\begin{array}{l}\text { Moderate } \\
\text { Impact on } \\
\text { Workload }\end{array}$ & $\begin{array}{l}\text { High Impact on } \\
\text { Workload }\end{array}$ & $\begin{array}{l}\text { Extreme } \\
\text { Impact on } \\
\text { Workload }\end{array}$ \\
\hline $\begin{array}{l}\text { 1.ADL - Self/Minimal } \\
\text { Care }\end{array}$ & 0 & 0 & 0 & 0 & 0 & 0 \\
\hline 2.ADL - Partial Care & 0 & 0 & 0 & 0 & 0 & 0 \\
\hline $\begin{array}{l}\text { 3.ADL - Complete } \\
\text { Care }\end{array}$ & 0 & 0 & 0 & 0 & 0 & 0 \\
\hline 4.ADL - Rehabilitative & 0 & 0 & 0 & 0 & 0 & 0 \\
\hline $\begin{array}{l}\text { 5.ADL Assistance - 2- } \\
3 \text { Caregivers }\end{array}$ & 0 & 0 & 0 & 0 & 0 & 0 \\
\hline $\begin{array}{l}\text { 6.ADL-Assistance-4 or } \\
\text { More Caregivers }\end{array}$ & 0 & 0 & 0 & 0 & 0 & 0 \\
\hline $\begin{array}{l}\text { 7.Communication } \\
\text { Support }\end{array}$ & 0 & 0 & 0 & 0 & 0 & 0 \\
\hline 8.Cognitive Support & 0 & 0 & 0 & 0 & 0 & 0 \\
\hline $\begin{array}{l}\text { 9.Behavior/Emotional } \\
\text { Management }\end{array}$ & 0 & 0 & 0 & 0 & 0 & 0 \\
\hline $\begin{array}{l}\text { 10.Behavior/Emotional } \\
\text { Mgmt - q } 1 \text { Hour }\end{array}$ & 0 & 0 & 0 & 0 & 0 & 0 \\
\hline $\begin{array}{l}\text { 11.Safety } \\
\text { Management - q } 2 \\
\text { Hours }\end{array}$ & 0 & 0 & 0 & 0 & 0 & 0 \\
\hline $\begin{array}{l}\text { 12.Safety } \\
\text { Management - q } 30 \\
\text { Minutes }\end{array}$ & 0 & 0 & 0 & 0 & 0 & 0 \\
\hline $\begin{array}{l}\text { 13.Isolation } \\
\text { Precautions } \\
\text { (Transmission-Based) }\end{array}$ & 0 & 0 & 0 & 0 & 0 & 0 \\
\hline $\begin{array}{l}\text { 14.Physiological } \\
\text { Assessment - q } 4 \\
\text { Hours }\end{array}$ & 0 & 0 & 0 & 0 & 0 & 0 \\
\hline $\begin{array}{l}\text { 15.Physiological } \\
\text { Assessment - q } 2 \\
\text { Hours }\end{array}$ & 0 & 0 & 0 & 0 & 0 & 0 \\
\hline
\end{tabular}




\begin{tabular}{|c|c|c|c|c|c|c|}
\hline \multicolumn{7}{|c|}{ Nurse Workload Study<br> } \\
\hline $\begin{array}{l}\text { 16.Physiological } \\
\text { Assessment - q } 1 \\
\text { Hour }\end{array}$ & 0 & 0 & 0 & 0 & 0 & 0 \\
\hline $\begin{array}{l}\text { 17.Physiological } \\
\text { Assessment - } 30 \\
\text { minutes }\end{array}$ & 0 & 0 & 0 & 0 & 0 & 0 \\
\hline $\begin{array}{l}\text { 18. Medication } \\
\text { Preparation >= } 20 \\
\text { Minutes }\end{array}$ & 0 & 0 & 0 & 0 & 0 & 0 \\
\hline $\begin{array}{l}\text { 19.Wound/Injury } \\
\text { Management }\end{array}$ & 0 & 0 & 0 & 0 & 0 & 0 \\
\hline $\begin{array}{l}\text { 20.Wound/Injury Mgmt } \\
>=30 \text { Minutes }\end{array}$ & 0 & 0 & 0 & 0 & 0 & 0 \\
\hline $\begin{array}{l}\text { 21. Healthcare Mgmt } \\
\text { Education }>=1 \text { Hour }\end{array}$ & 0 & 0 & 0 & 0 & 0 & 0 \\
\hline 46.PtOb/confusion & 0 & 0 & 0 & 0 & 0 & 0 \\
\hline $\begin{array}{l}\text { 47.PtOb/drug or etoh } \\
\text { withdrawal }\end{array}$ & 0 & 0 & 0 & 0 & 0 & 0 \\
\hline 48.PtOb/mental illness & 0 & 0 & 0 & 0 & 0 & 0 \\
\hline $\begin{array}{l}50 . \mathrm{PtOb} / \text { suicide } \\
\text { precautions }\end{array}$ & 0 & 0 & 0 & 0 & 0 & 0 \\
\hline 70.PtOb/fall risk & 0 & 0 & 0 & 0 & 0 & 0 \\
\hline
\end{tabular}

7. Are there any other factors, which are not included in the patient acuity indicators above that affect your workload? if there are, please list them in order.

Factor-1

Factor-2

Factor-3

Factor-4

Factor-5

Thank you very much for completing this survey! 


\section{Appendix C}

\section{Python Code}

Here is python code we used to run our models. For each model, indicated parts are encoded.

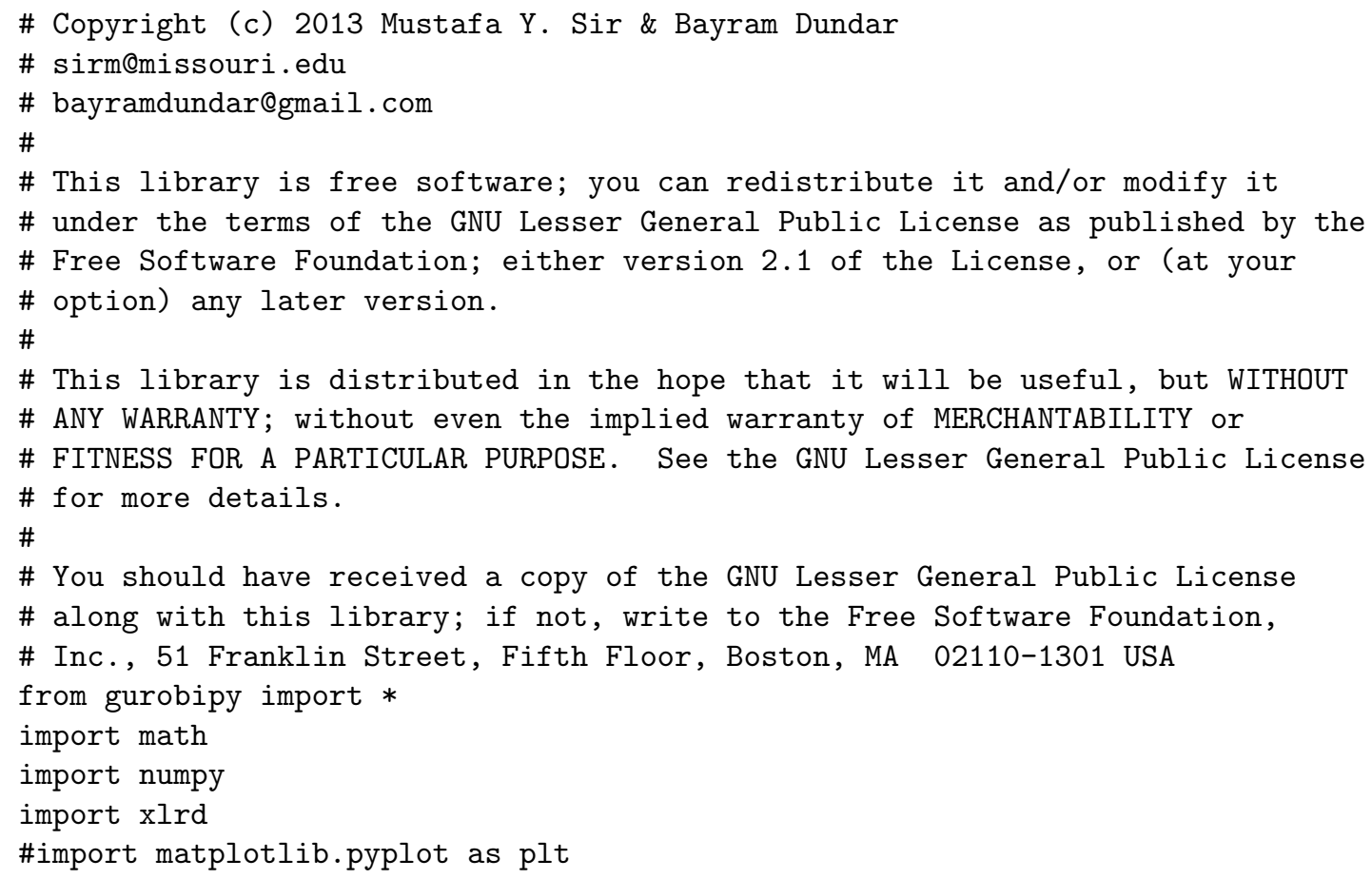




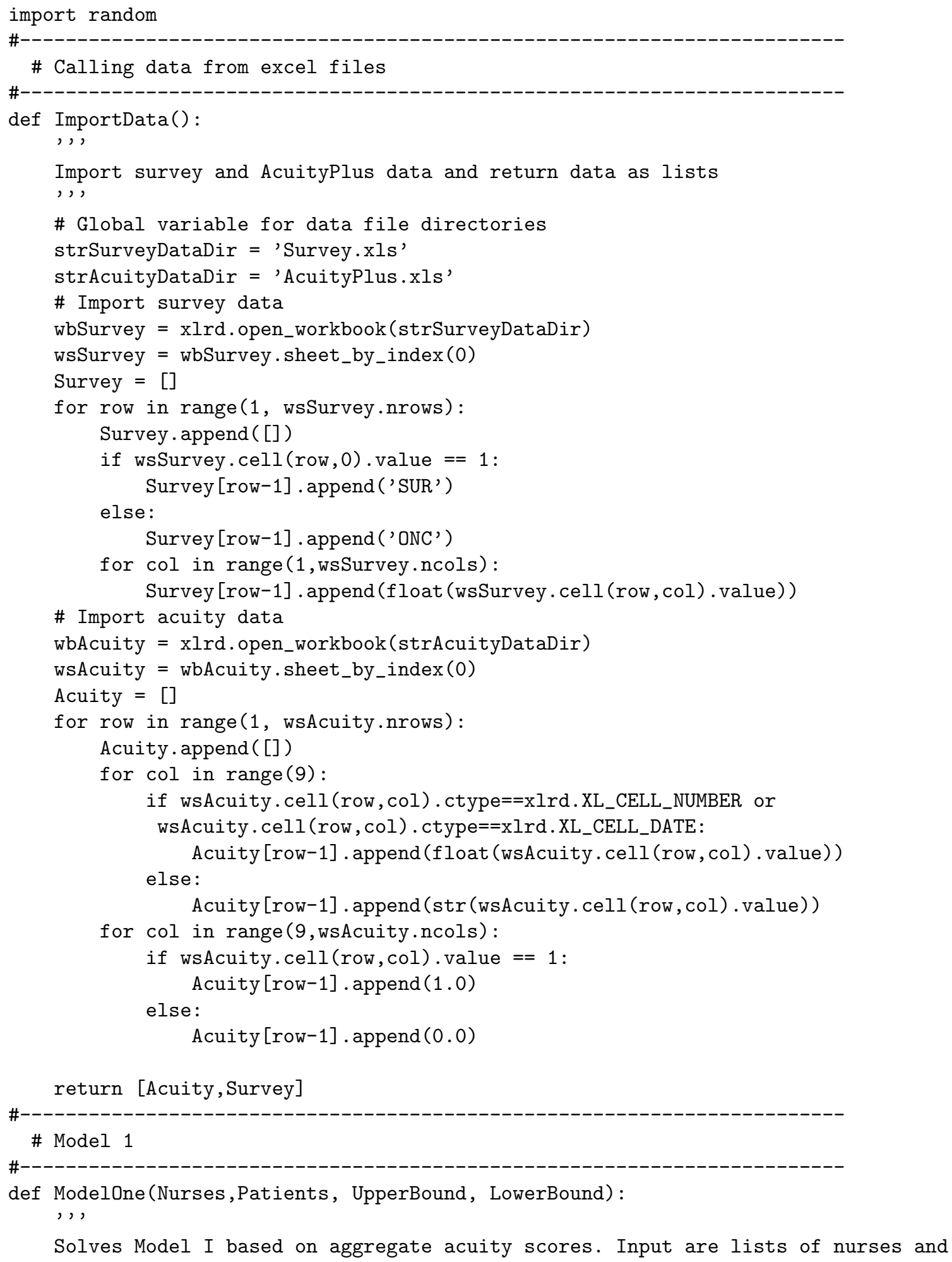




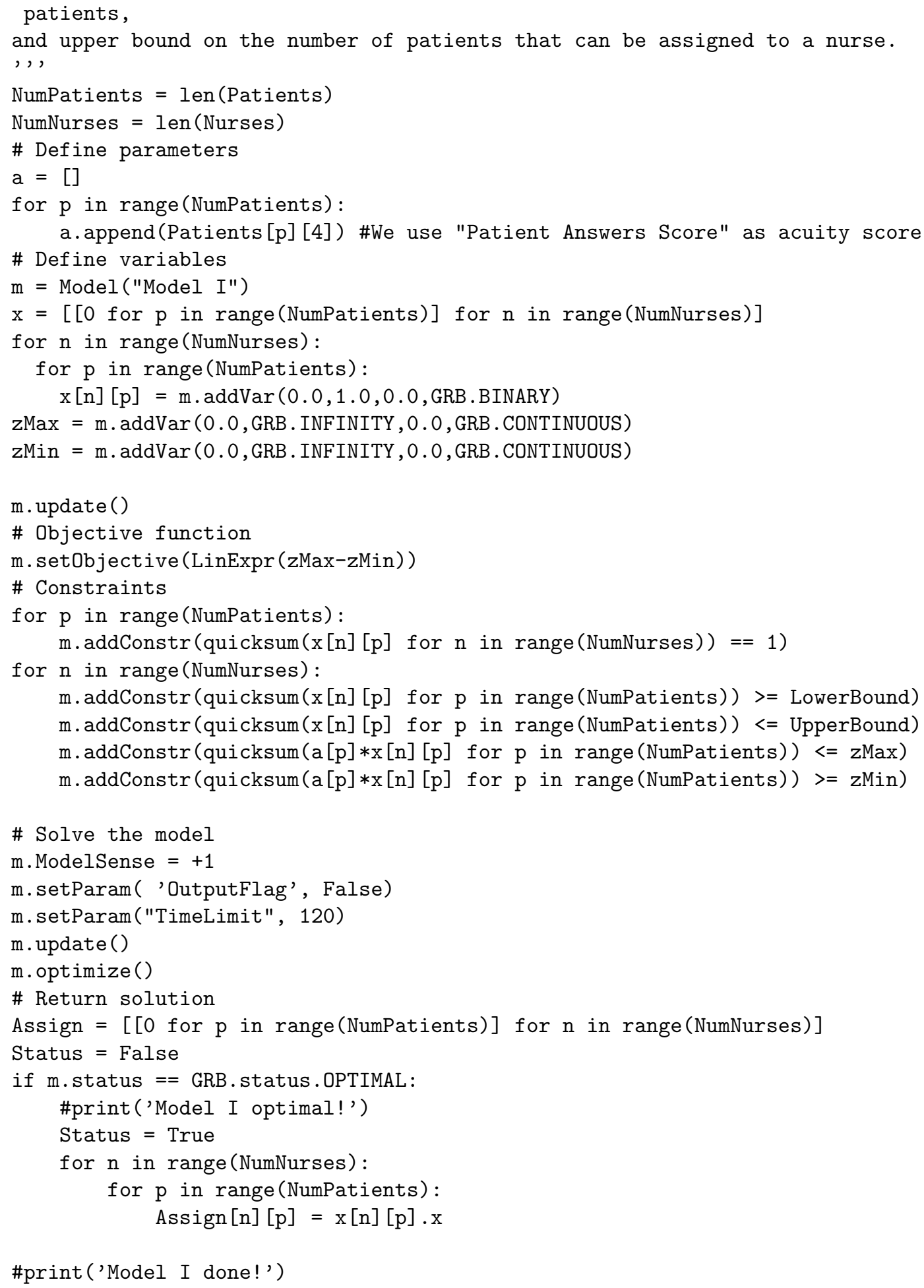


return [Status, Assign]

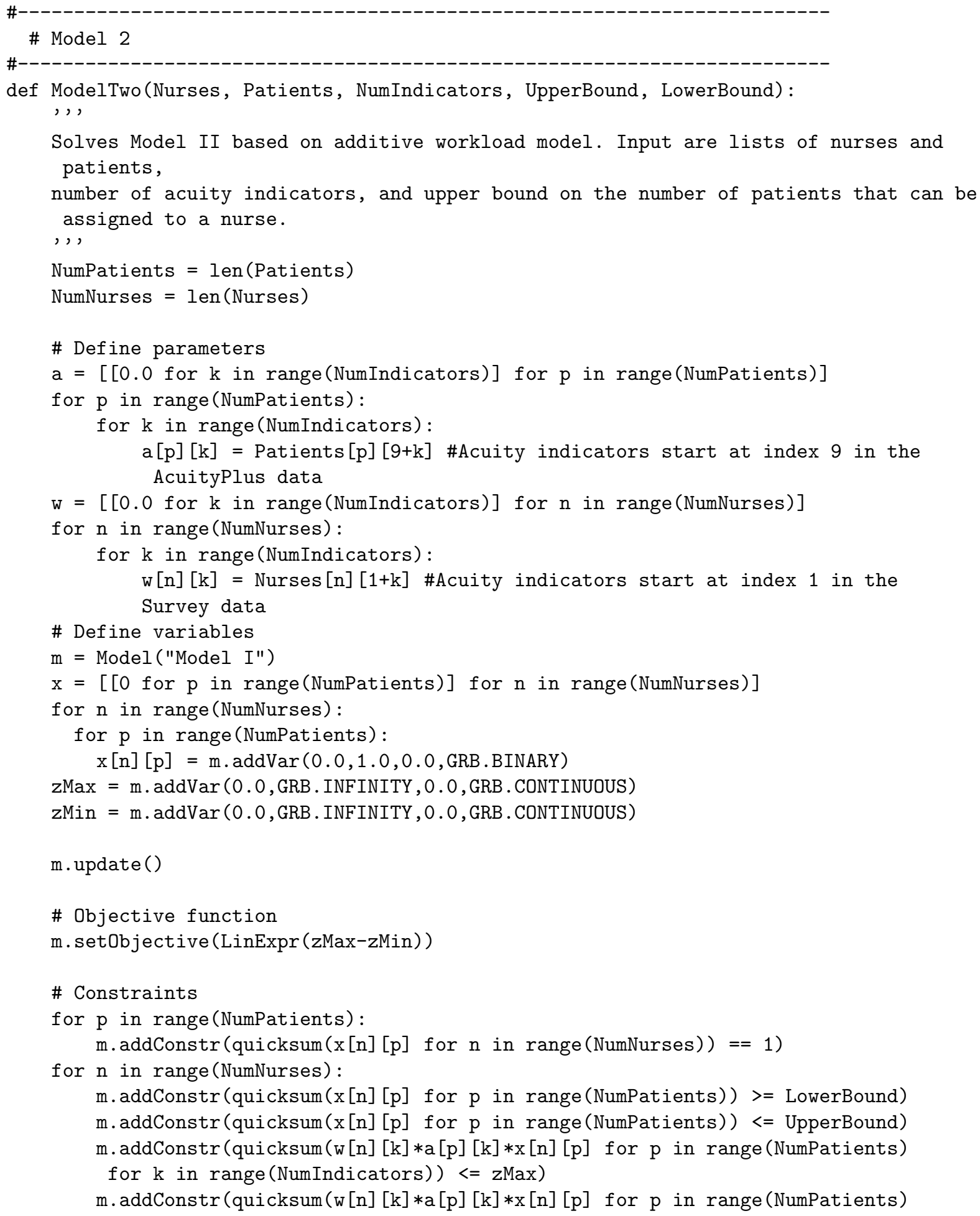


for $\mathrm{k}$ in range(NumIndicators)) >= zMin)

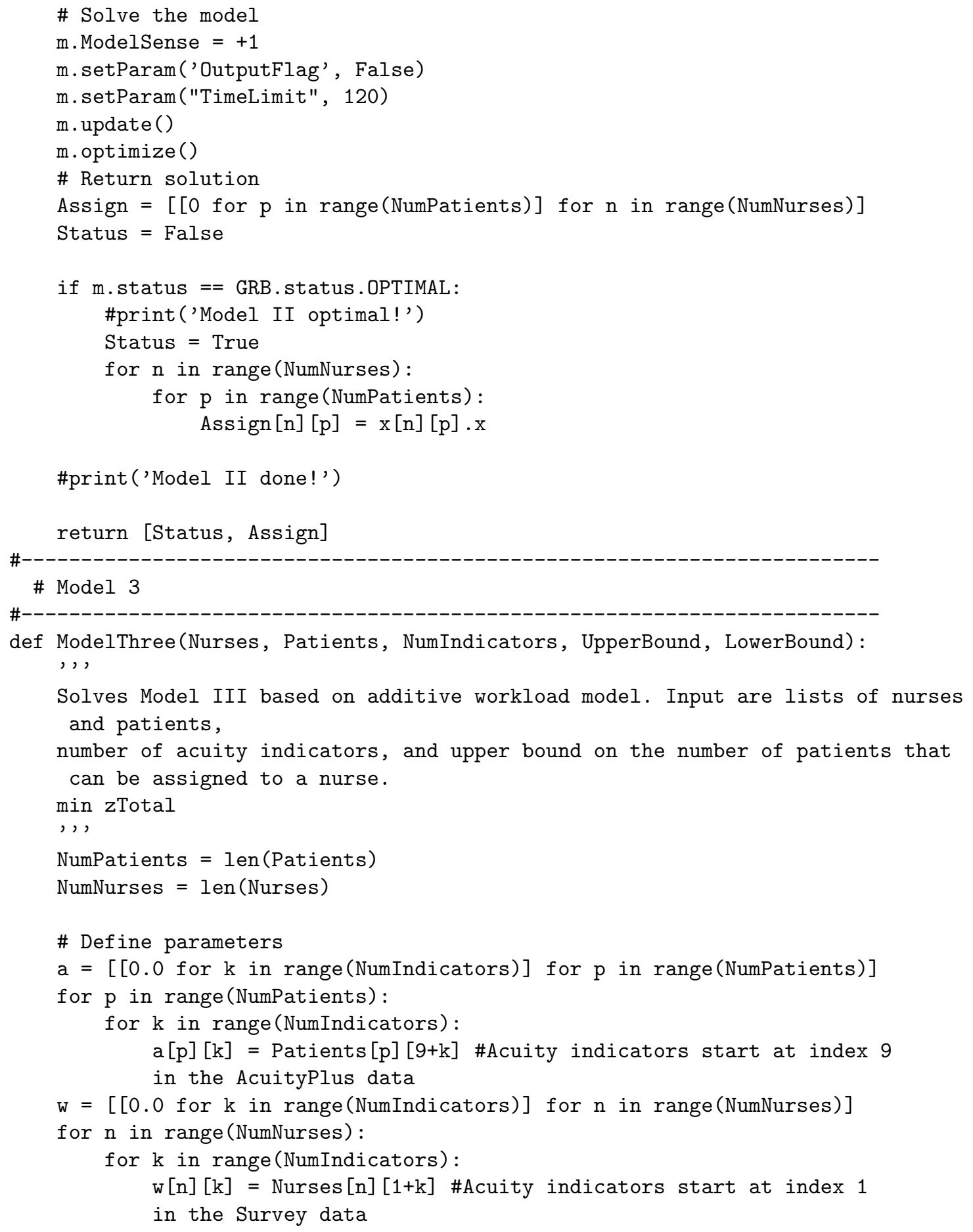




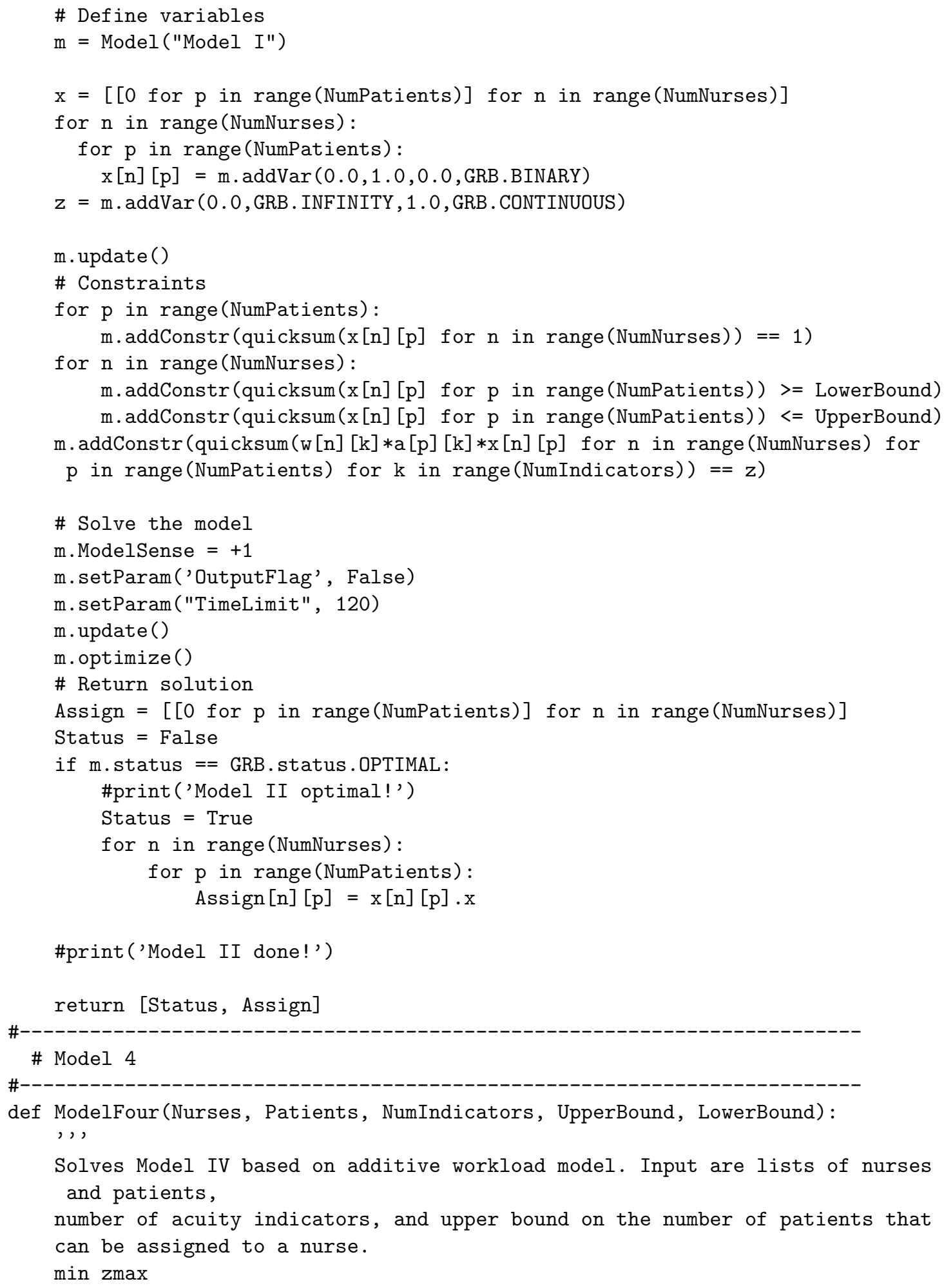




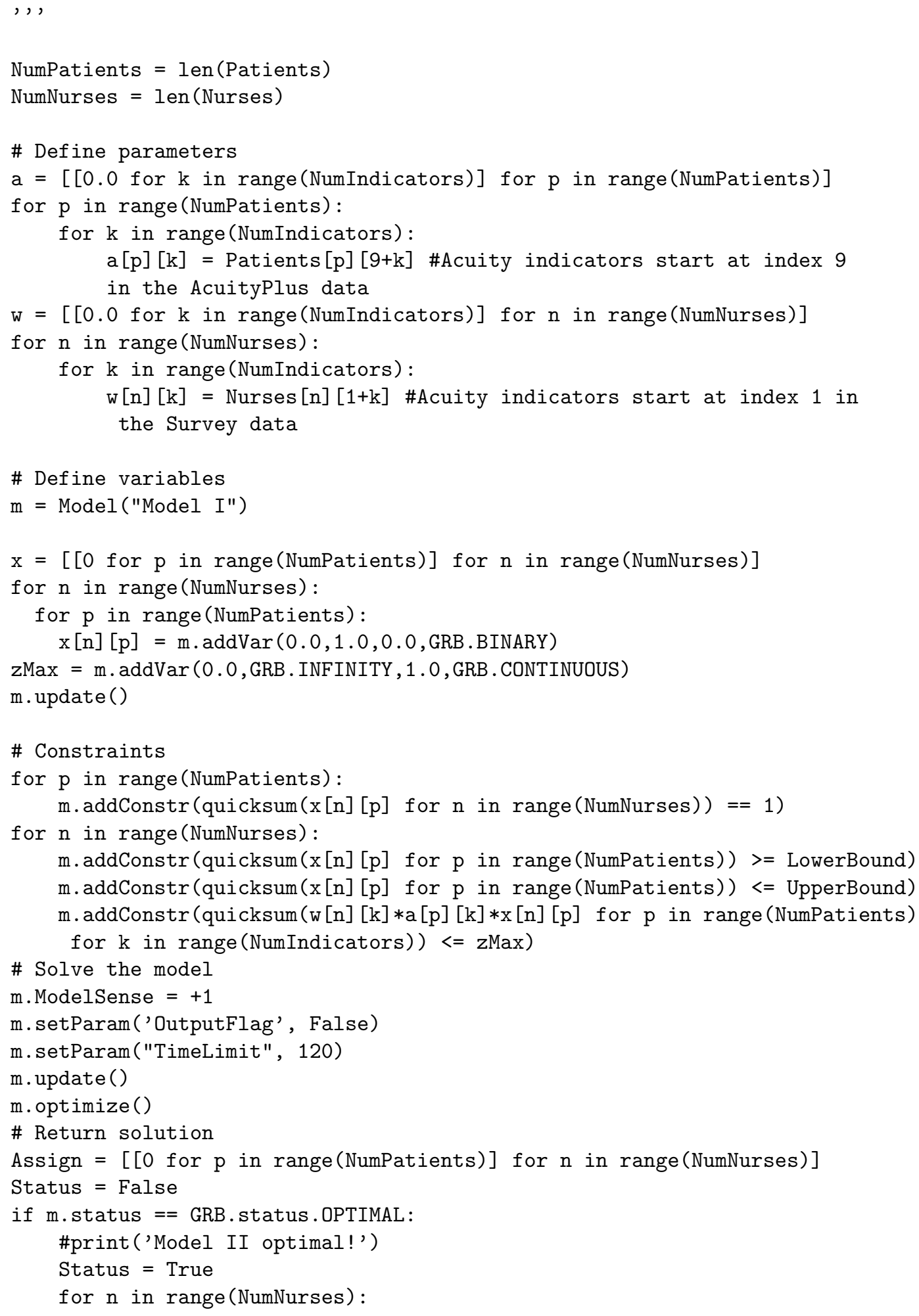




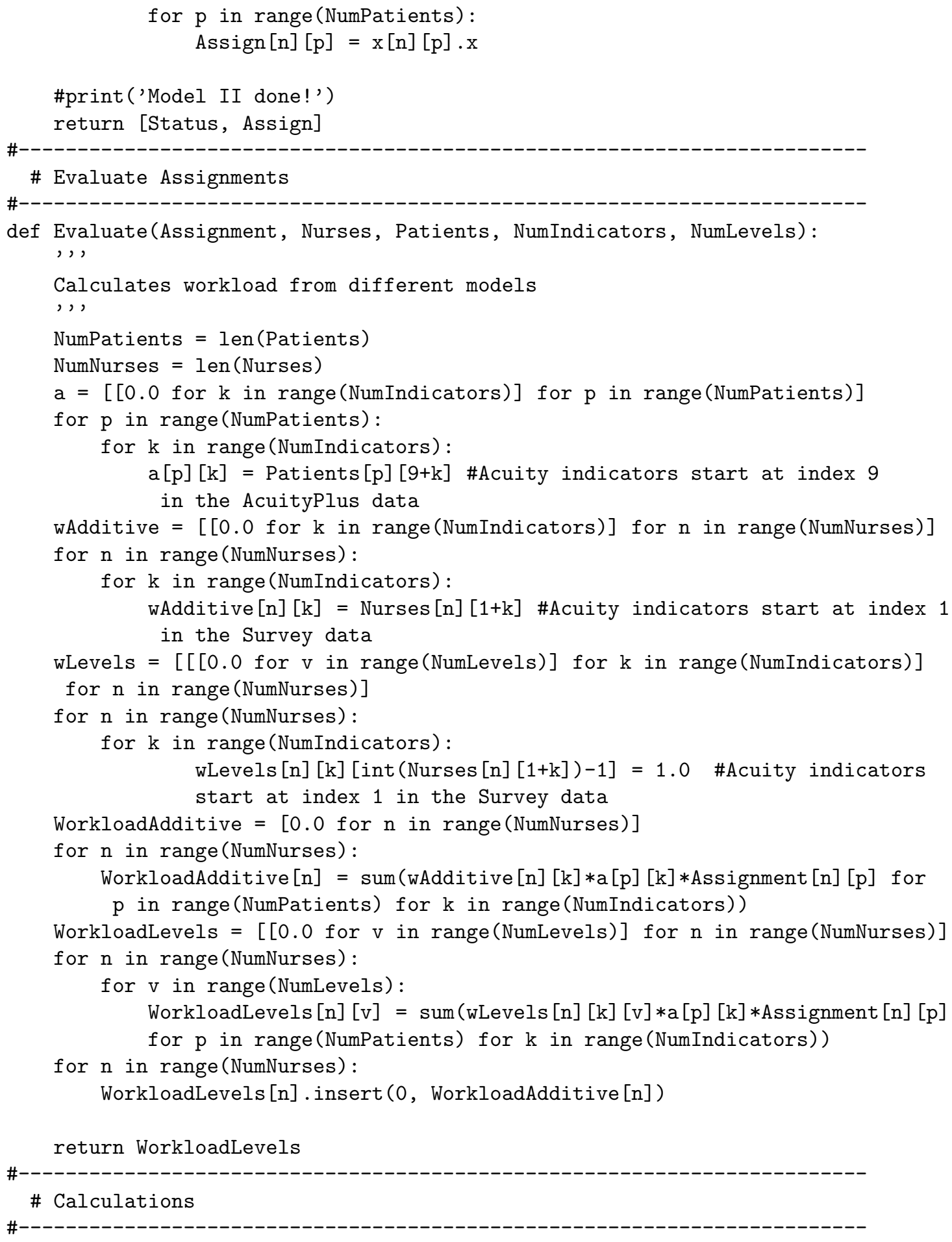




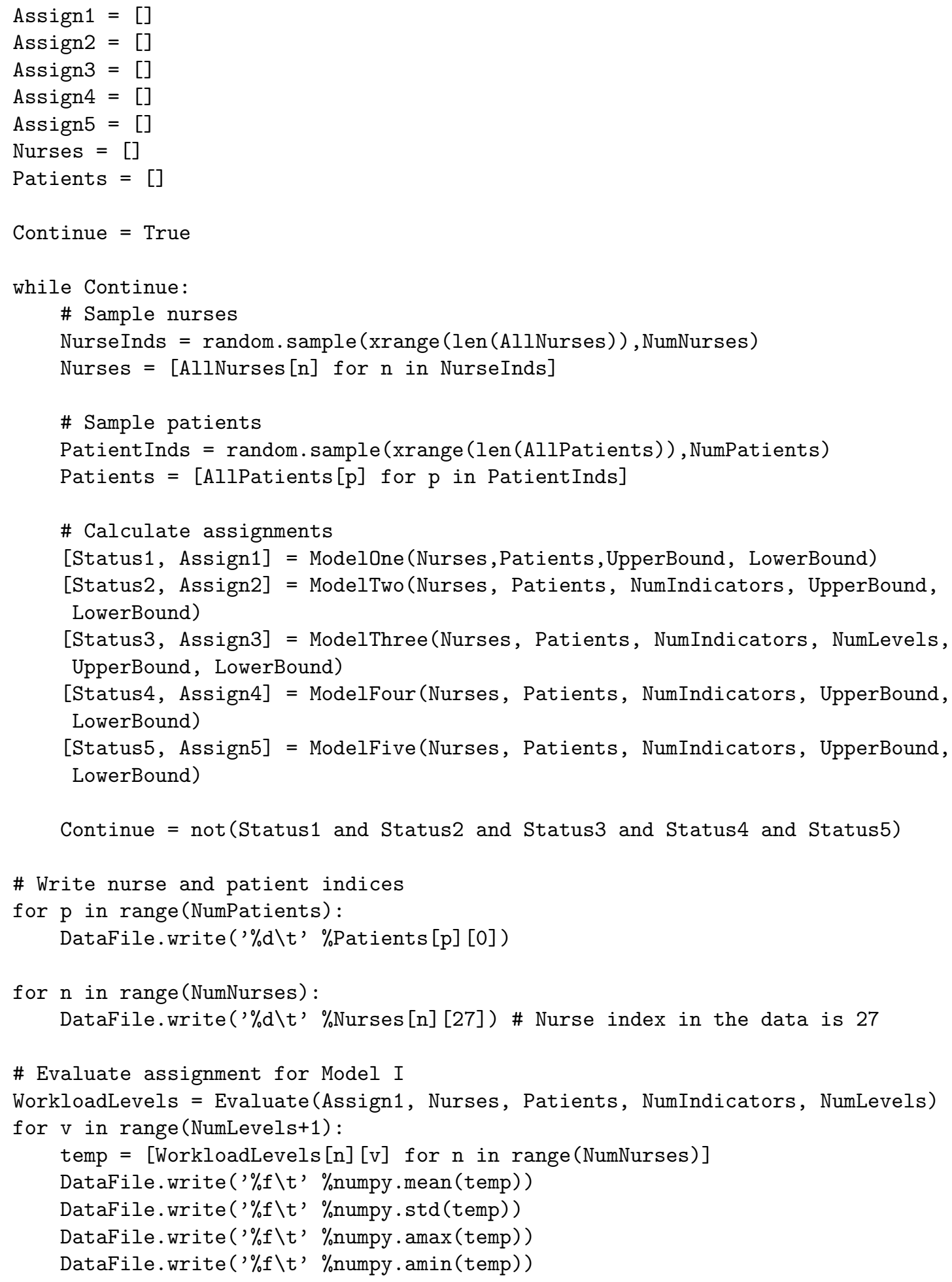


\# Evaluate assignment for Model II

WorkloadLevels = Evaluate(Assign2, Nurses, Patients, NumIndicators, NumLevels)

for $\mathrm{v}$ in range (NumLevels +1 ):

temp $=$ [WorkloadLevels $[\mathrm{n}][\mathrm{v}]$ for $\mathrm{n}$ in range(NumNurses)]

DataFile.write ( $\%$ f $\backslash t, \%$ numpy.mean (temp))

DataFile.write ( $\%$ f $\backslash$ t' $\%$ numpy.std (temp))

DataFile.write ( $\%$ f $\backslash t$, \%numpy. amax (temp))

DataFile.write ( $\% f \backslash t$, \%numpy. amin (temp))

\# Evaluate assignment for Model III

WorkloadLevels = Evaluate(Assign3, Nurses, Patients, NumIndicators, NumLevels)

for $\mathrm{V}$ in range (NumLevels+1):

temp $=$ [WorkloadLevels $[\mathrm{n}][\mathrm{v}]$ for $\mathrm{n}$ in range(NumNurses)]

DataFile.write ( $\%$ f $\backslash$ t' \%numpy.mean (temp))

DataFile.write ( $\%$ f $\backslash$ t' $\%$ numpy.std (temp))

DataFile.write ( $\%$ f $\backslash t$ ' \%numpy. amax (temp))

DataFile.write ( $\%$ f $\backslash t$ ' \%umpy.amin(temp))

\# Evaluate assignment for Model IV

WorkloadLevels = Evaluate(Assign4, Nurses, Patients, NumIndicators, NumLevels)

for $\mathrm{v}$ in range (NumLevels+1):

temp $=$ [WorkloadLevels $[\mathrm{n}][\mathrm{v}]$ for $\mathrm{n}$ in range(NumNurses)]

DataFile.write ( $\%$ f $\backslash$ t' \%numpy.mean (temp))

DataFile.write ( $\%$ f $\backslash$ ' $\%$ numpy.std (temp))

DataFile.write ( $\%$ f $\backslash t, \%$ numpy. amax (temp))

DataFile.write ( \% $\%$ t' \%numpy. amin(temp))

\# Evaluate assignment for Model V

WorkloadLevels = Evaluate(Assign5, Nurses, Patients, NumIndicators, NumLevels)

for $\mathrm{v}$ in range (NumLevels +1$)$ :

temp $=$ [WorkloadLevels [n] [v] for $\mathrm{n}$ in range(NumNurses)]

DataFile.write ( $\%$ f $\backslash t$ ' \% numpy.mean (temp))

DataFile.write ( $\% f \backslash t$ ' \%numpy.std(temp))

DataFile.write ( $\%$ f $\backslash t$ ' \%numpy. $\operatorname{amax}($ temp))

DataFile.write ( $\%$ f $\backslash t$ ' \%numpy. $\operatorname{amin}($ temp))

DataFile.write(' $\backslash \mathrm{n}$ ')

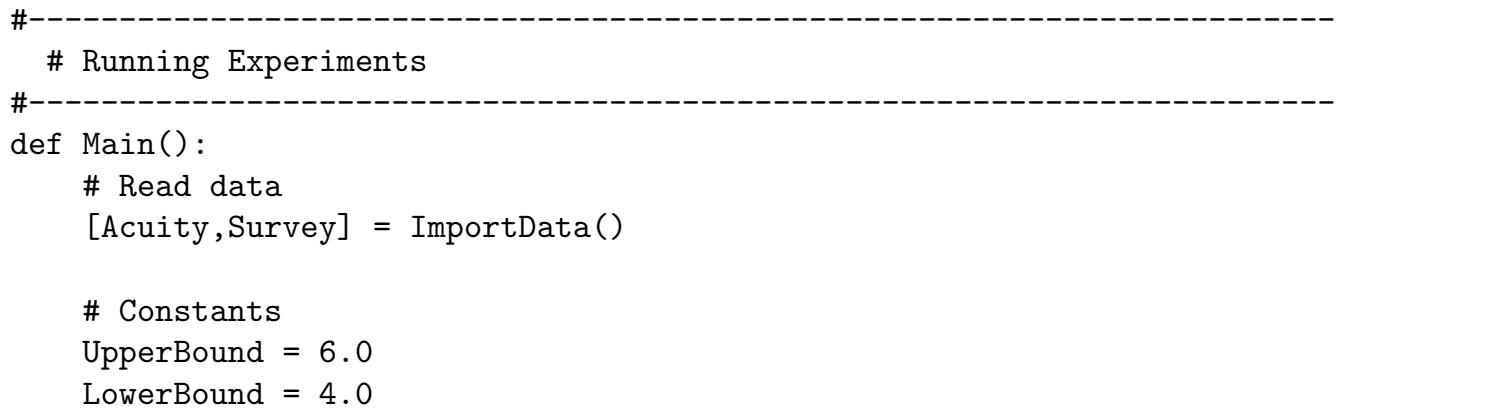


NumIndicators $=26$

NumLevels $=6$

NumPatients $=30$

NumNurses $=6$

NumExperiments $=100$

\# Oncology Experiments

PatientsONC $=\left[p\right.$ for $p$ in Acuity if $p[1]=={ }^{\prime}$ ONC' $]$

NursesONC $=\left[\mathrm{n}\right.$ for $\mathrm{n}$ in Survey if $\left.\mathrm{n}[0]=={ }^{\prime} \mathrm{ONC} \mathrm{C}^{\prime}\right]$

\# Write data to a file

DataFile = open('ResultsONC.txt', 'w')

DataFile.write('Exp Ind' + '\t')

for $\mathrm{n}$ in range(NumNurses):

DataFile.write('Nurse \%d\t' \%n)

for $p$ in range(NumPatients):

DataFile.write ('Patient \%d\t' \%p)

for $v$ in range (NumLevels +1 ):

DataFile.write('M1 WL\%d Mean \t' $\% \mathrm{v}$ )

DataFile.write ('M1 WL\%d Std \t' $\% \mathrm{v}$ )

DataFile.write('M1 WL\%d Max \t' $\% \mathrm{v}$ )

DataFile.write ('M1 WL\%d Min \t' $\% v$ )

for $v$ in range (NumLevels +1 ):

DataFile.write('M2 WL\%d Mean \t' $\% v$ )

DataFile.write ('M2 WL\%d Std \t' $\% \mathrm{v}$ )

DataFile.write ('M2 WL\%d Max \t' $\% v$ )

DataFile.write ('M2 WL\%d Min \t' $\% v$ )

for $v$ in range (NumLevels +1 ):

DataFile.write('M3 WL\%d Mean \t' $\% v$ )

DataFile.write ('M3 WL\%d Std \t' $\% \mathrm{v}$ )

DataFile.write ('M3 WL\%d Max \t' $\% v$ )

DataFile.write ('M3 WL\%d Min \t' $\% v$ )

for $\mathrm{v}$ in range (NumLevels+1):

DataFile.write ('M4 WL\%d Mean \t' $\% \mathrm{v}$ )

DataFile.write ('M4 WL\%d Std \t' $\% \mathrm{v}$ )

DataFile.write ('M4 WL\%d Max \t' $\% v$ )

DataFile.write ('M4 WL\%d Min \t' $\% \mathrm{v}$ )

for $v$ in range (NumLevels +1 ):

DataFile.write('M5 WL\%d Mean \t' $\% \mathrm{v}$ )

DataFile.write('M5 WL $\%$ d Std \t' $\% \mathrm{v}$ )

DataFile.write ('M5 WL\%d Max \t' $\% v$ )

DataFile.write ('M5 WL\%d Min \t' $\% \mathrm{v}$ )

DataFile.write(' $\backslash \mathrm{n}$ ')

\# Experiment loop

for $e$ in range(NumExperiments): 


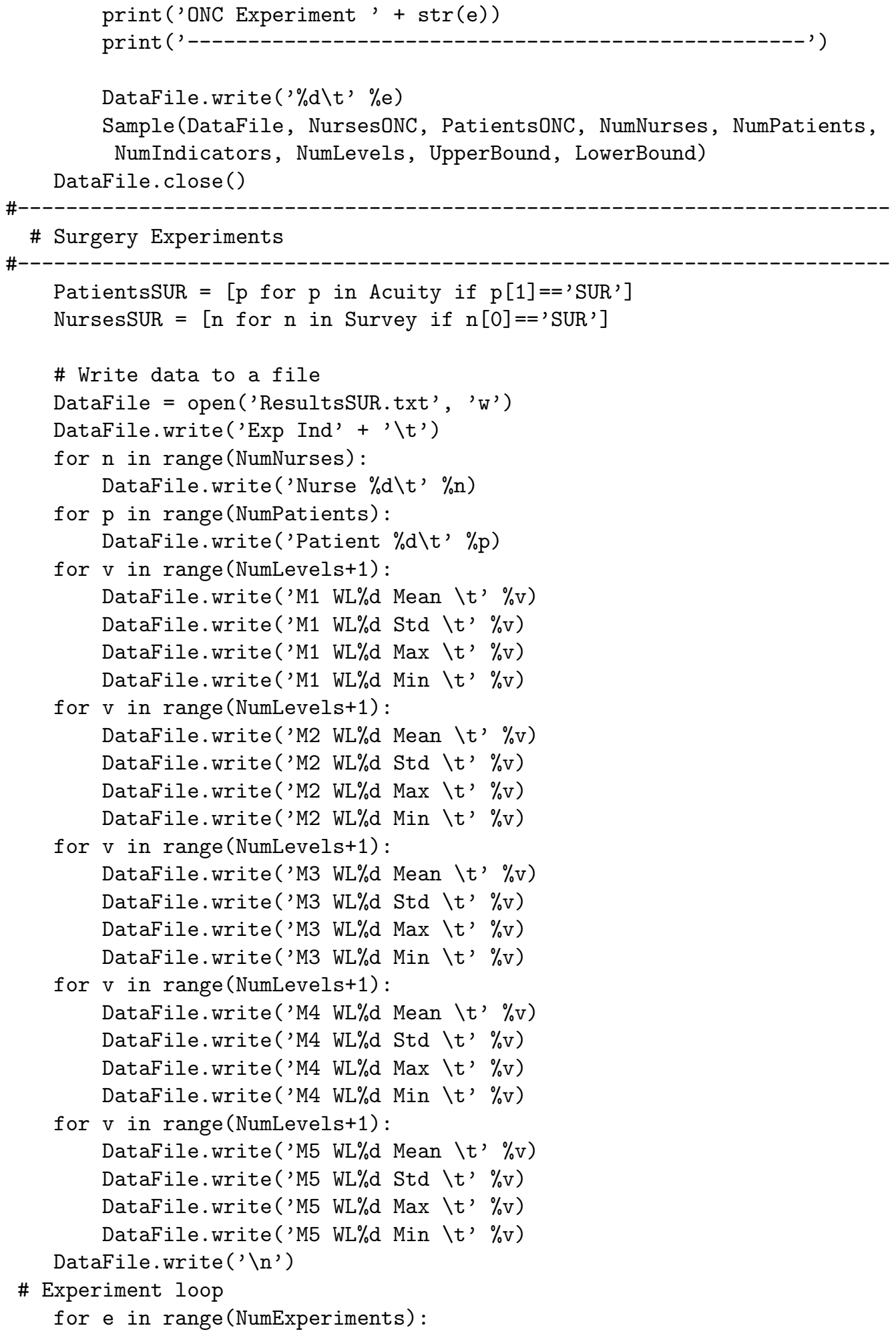


print ('SUR Experiment' $+\operatorname{str}(e))$

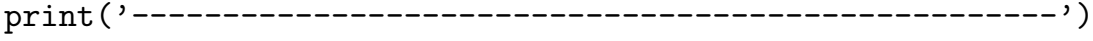

DataFile.write ( $\% \mathrm{~d} \backslash t, \% e)$

Sample(DataFile, NursesSUR, PatientsSUR, NumNurses, NumPatients, NumIndicators, NumLevels, UpperBound, LowerBound)

DataFile.close()

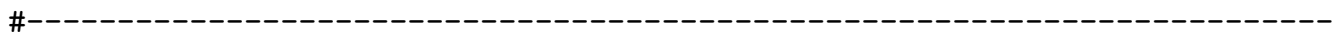

\#

if _-_name_-_ == "-_main_-- :

$\operatorname{Main}($ ) 


\section{Bibliography}

Aickelin, U. \& Li, J. (2007), 'An estimation of distribution algorithm for nurse scheduling', Annals of Operations Research 155(1), 289-309.

American Nurses Association (2011), '2011 ana health and safety survey'.

URL: /http://www.nursingworld.org/

Battisto, D., Pak, R., Vander Wood, M. A. \& Pilcher, J. J. (2009), 'Using a task analysis to describe nursing work in acute care patient environments', Journal of Nursing Administration 39(12), 537547.

Bilgin, B., De Causmaecker, P., Rossie, B. \& Berghe, G. V. (2012), 'Local search neighbourhoods for dealing with a novel nurse rostering model', Annals of Operations Research 194(1), 33-57.

Biviano, M., Dall, T., Grover, A., Tise, S., Fritz, M. \& Spencer, W. (2005), 'Projected supply, demand, and shortages of registered nurses', FORECASTERS p. 157.

Brennan, C. W., Daly, B. J., Dawson, N. V., Higgins, P. A., Jones, K. R., Madigan, E. \& Van Der Meulen, J. (2012), 'The oncology acuity tool: A reliable, valid method for measuring patient acuity for nurse assignment decisions', Journal of nursing measurement 20(3), 155-185.

Chomeya, R. (2010), 'Quality of psychology test between likert scale 5 and 6 points', Journal of Social Sciences 6(3), 399-403.

de Véricourt, F. \& Jennings, O. B. (2011), 'Nurse staffing in medical units: A queueing perspective', Operations research $\mathbf{5 9}(6), 1320-1331$.

Ebright, P. R., Patterson, E. S., Chalko, B. A. \& Render, M. L. (2003), 'Understanding the complexity of registered nurse work in acute care settings', Journal of Nursing Administration 33(12), 630638.

Graf, C. M., Millar, S., Feilteau, C., Coakley, P. J. \& Erickson, J. I. (2003), 'Patients' needs for nursing care: beyond staffing ratios', Journal of Nursing Administration 33(2), 76-81.

Gurobi Optimization (2013), 'Gurobi optimizer reference manual'.

URL: http://www.gurobi.com 
Harper, K. \& McCully, C. (2007), 'Acuity systems dialogue and patient classification system essentials', Nursing administration quarterly 31(4), 284-299.

Hertz, A. \& Lahrichi, N. (2008), 'A patient assignment algorithm for home care services', Journal of the Operational Research Society 60(4), 481-495.

Kalisch, B. J. \& Aebersold, M. (2010), 'Interruptions and multitasking in nursing care', Joint Commission Journal on Quality and Patient Safety 36(3), 126-132.

Kortbeek, N., Braaksma, A., Burger, C., Bakker, P. \& Boucherie, R. (2012), 'Flexible nurse staffing based on hourly bed census predictions'.

Lin, R.-C., Sir, M. Y., Sisikoglu, E., Pasupathy, K. \& Steege, L. M. (2013), 'Optimal nurse scheduling based on quantitative models of work-related fatigue', IIE Transactions on Healthcare Systems Engineering 3(1), 23-38.

Maenhout, B. \& Vanhoucke, M. (2010), 'Branching strategies in a branch-and-price approach for a multiple objective nurse scheduling problem', Journal of Scheduling 13(1), 77-93.

Maenhout, B. \& Vanhoucke, M. (2013), 'An integrated nurse staffing and scheduling analysis for longer-term nursing staff allocation problems', Omega 41(2), 485-499.

Minitab Inc. (2009), 'Minitab 16 statistical software'.

URL: http://www.minitab.com

Mullinax, C., Lawley, M. et al. (2002), 'Assigning patients to nurses in neonatal intensive care', Journal of the Operational Research Society 53(1), 25-35.

Patterson, E. S., Ebright, P. R. \& Saleem, J. J. (2011), 'Investigating stacking: How do registered nurses prioritize their activities in real-time?', International Journal of Industrial Ergonomics 41(4), 389-393.

Potter, P., Boxerman, S., Wolf, L., Marshall, J., Grayson, D., Sledge, J. \& Evanoff, B. (2004), 'Mapping the nursing process: a new approach for understanding the work of nursing', Journal of Nursing Administration 34(2), 101-109.

Punnakitikashem, P., Rosenberber, J. M. \& Buckley-Behan, D. F. (2013), 'A stochastic programming approach for integrated nurse staffing and assignment', IIE Transactions (just-accepted).

Python Software Foundation (2013), 'Python programming language'.

URL: /http://www.python.org/

QuadraMed (2008), AcuityPlus System Inpatient Methodology, Classification Indicator Education for University Hospital Columbia, MO, 1 edn, QuadraMed Corporation, Chicago, IL.

Rais, A. \& Viana, A. (2011), 'Operations research in healthcare: a survey', International Transactions in Operational Research 18(1), 1-31.

Sundaramoorthi, D., Chen, V. C., Rosenberger, J. M., Kim, S. B. \& Buckley-Behan, D. F. (2009), 'A data-integrated simulation model to evaluate nurse-patient assignments', Health care management science 12(3), 252-268. 
Sundaramoorthi, D., Chen, V. C., Rosenberger, J. M., Kim, S. B. \& Buckley-Behan, D. F. (2010), 'A data-integrated simulation-based optimization for assigning nurses to patient admissions', Health care management science 13(3), 210-221.

Wolf, L. D., Potter, P., Sledge, J. A., Boxerman, S. B., Grayson, D. \& Evanoff, B. (2006), 'Describing nurses' work: combining quantitative and qualitative analysis', Human Factors: The Journal of the Human Factors and Ergonomics Society 48(1), 5-14.

Wright, P. D., Bretthauer, K. M. \& Côté, M. J. (2006), 'Reexamining the nurse scheduling problem: Staffing ratios and nursing shortages*', Decision Sciences 37(1), 39-70.

Yankovic, N. \& Green, L. V. (2008), 'A queueing model for nurse staffing', Under review at Operations Research 7.

Yankovic, N. \& Green, L. V. (2011), 'Identifying good nursing levels: A queuing approach', Operations research 59(4), 942-955. 